\title{
Discrete element modeling of permeability evolution during progressive failure of a low-permeable rock under triaxial compression
}

\author{
Lingran Zhang ${ }^{1}$, Luc Scholtès ${ }^{*}$, Frédéric Victor Donzé \\ lingrano.zhang@gmail.com \\ luc.scholtes@uca.fr \\ frederic.donze@univ-grenoble-alpes.fr
}

\author{
${ }^{1}$ Univ. Grenoble Alpes, Univ. Savoie Mont Blanc, CNRS, IRD, IFSTTAR, ISTerre, 38000 Grenoble, France \\ ${ }^{2}$ Univ. Clermont Auvergne, CNRS, IRD, OPGC, Laboratoire Magmas et Volcans, 63000 Clermont-Ferrand, France
}

\begin{abstract}
Understanding permeability evolution caused by the nucleation, propagation and coalescence of cracks enables to better assess fluid migration in the vicinity of underground excavations, boreholes or reservoirs. In this study, we propose a three-dimensional approach combining a bonded particle model and a dual-permeability pore network model to investigate the crack permeability behavior of low-permeable rocks. First, we verify the performances of the numerical scheme by comparing its predictions to analytical permeability solutions for microcracked and fractured porous samples respectively. Then, we simulate a triaxial compression test on an argillaceous rock sample with periodic permeability measurements. The model is able to reproduce the stressstrain-permeability evolution observed experimentally, from the early stage of microcracking up to the residual post-failure state: i) permeability does not change significantly before reaching the crack damage threshold, ii) permeability increases by several orders of magnitude after failure due to the appearance of a discrete shear band across the sample. The good agreement between the numerical results and the experimental observations confirms the relevance of the proposed approach to simulate the crack permeability behavior of low permeable rocks during their progressive failure. Based on this result, we simulate triaxial compression tests under different confining pressures to propose relationships between post-failure permeability and residual mean stress.
\end{abstract}

Keywords Low permeable rock, Permeability, Cracks, Failure, Discrete Element Method 


\section{List of Symbols}

$R_{a} \quad$ Radius of the particle a

$D_{a b}^{0} \quad$ Initial distance between the centroids of two interacting particles

$\gamma_{\text {int }} \quad$ Interaction range coefficient

$F_{n} \quad$ Normal interaction force

$\Delta D \quad$ Normal relative displacement

$k_{n} \quad$ Normal stiffness

$E_{e q} \quad$ Local equivalent Young modulus

$F_{n, \max }$ Maximum admissible tensile force

$t \quad$ Tensile strength of the interparticle bond

$A_{\text {int }} \quad$ Interacting surface area between particles a and $\mathrm{b}$

$F_{S} \quad$ Shear interaction force

$\Delta u_{s} \quad$ Relative incremental tangential displacement

$k_{s} \quad$ Shear stiffness

$\delta \quad$ Stiffness ratio

$F_{s, \max } \quad$ Maximum admissible shear force

$c \quad$ Local cohesion

$\varphi \quad$ Local frictional angle

$V_{i}^{f} \quad$ Fluid volume contained in pore $i$

$q_{i j} \quad$ Fluid flux between pore $i$ and $j$

$k_{i j} \quad$ Local conductance linking pore $i$ and $j$

$p_{i} \quad$ Fluid pressures in pore $i$

$\alpha \quad$ Scaling factor

$S_{i j}^{f} \quad$ Pore-throat's cross-sectional area

$R_{i j}^{h} \quad$ Hydraulic radius

$L_{i j} \quad$ Length of the interpore throat $i j$

$\Omega_{i} \quad$ Domain defined by tetrahedron $i$

$\Omega_{i j} \quad$ Union of tetrahedra $i$ and $j$

$\Theta_{i j} \quad$ Part of $\Omega_{i j}$ occupied by the fluid phase

$P_{i} \quad$ Voronoi dual (weighted center) of tetrahedron $i$

$h \quad$ Crack's aperture $\mu \quad$ Dynamic viscosity of the fluid

$h_{r} \quad$ Residual aperture or aperture of pre-existing crack

$\Delta \mathrm{p} \quad$ Pressure difference

$Q \quad$ Volumetric flow rate at equilibrium

A Cross-sectional area of the sample

$L \quad$ Length of the sample

$k \quad$ Permeability of the sample

$\theta \quad$ Fracture orientation with respect to the $\mathrm{X}$ direction

$\chi \quad$ Fracture density defined in Hyman et al. (2018)

$k_{e} \quad$ Effective permeability defined in Hyman et al. (2018)

$k_{f} \quad$ Fracture permeability

$k_{m} \quad$ Permeability of the intact medium

$\beta \quad$ Orientation of pre-existing micro cracks

$\Delta \beta \quad$ Angle range

$P_{32} \quad$ Crack intensity

$P_{33} \quad$ Crack volume

$k x \quad$ Permeability measured for $\mathrm{X}$ direction flow

$\varepsilon_{1} \quad$ Axial strain

$\varepsilon_{1}^{\text {peak }} \quad$ Axial strain at the peak stress

$\sigma_{e} \quad$ Residual mean stress

$\sigma_{3} \quad$ Lateral confining pressure

$c_{f} \quad$ Fracture compressibility

$k_{\max } \quad$ Upper limit of the post-failure permeability

$E_{0} \quad$ Macroscopic Young modulus of the rock material

A Scaling parameter

$n_{\text {crack }}$ Number of cracks

$n_{\text {total }}$ Number of initial bonds

$\lambda$ Scaling parameter

$k_{\text {min }} \quad$ Lower limit of the post-peak permeability value 


\section{Introduction}

Stress disturbances in low permeable rocks induce the nucleation and propagation of cracks which largely control their hydraulic properties by forming preferential pathways for groundwater flows (Brace 1980; Guéguen et al. 1996). Assessing the crack permeability behavior of low permeable geological media subjected to stress alterations is thus essential in strategic geoengineering applications such as geologic storage of hazardous substances (Armand et al. 2014; Tsang et al. 2015), geological sequestration of greenhouse gases (Tsang et al, 2007; Lemieux, 2011), and energy resources extraction (Dahm et al. 2015, Cui et al. 2013, Han et al. 2015).

Stress-induced cracks nucleate and grow progressively until they coalesce to eventually form fractures at a larger-scale. The level of their interconnection directly controls the permeability of the medium they are embedded into (Sahimi 1994). If the cracks are not connected, the effective permeability of the medium is determined by the properties of the rock matrix. If the cracks are connected and form fractures that percolate throughout the medium, the effective permeability is determined by the fracture network properties. Stressinduced fractures can actually lead to major changes in the hydraulic properties of rocks over long distances (Zhang and Sanderson 1998; Bogdanov et al. 2003; Yazdi et al. 2011; Mitchell and Faulkner 2012; Chen et al. 2018; Hyman et al. 2018). This is especially true if the porosity of the rock matrix is low, since the permeability increase can be of several orders of magnitude as observed in granites (Souley et al. 2001; Chen et al. 2014), limestones (Selvadurai and Glowacki, 2017) and argillaceous rocks like claystones (Zhang 2016; Bossart et al. 2002; Armand et al. 2014) or shales (Matray et al. 2007; Carey et al. 2015).

Since the early studies of Brace $(1968 ; 1978)$ and Zoback and Byerlee (1975a; 1975b), many works have been conducted to assess permeability changes in low porosity rocks due to stress variations and a rather complete picture has been provided on how permeability depends on the deformation mechanisms. In such rocks, dilatancy is generally observed whether the material fails by brittle faulting or cataclastic flow under compressive loads. Unlike in porous rocks where grain crushing and pore collapse can produce drastic decrease of permeability (Zhu and Wong 1997), changes in the pore space of low porosity rocks are mostly due to stressinduced microcracking which tends to increase the overall permeability (Paterson and Wong 2005). For instance, Zoback and Byerlee (1975) have observed permeability increase by a factor of $\sim 3$ in granite at $\sim 80 \%$ of the peak loads in the brittle faulting regime, while Stormont and Daemen (1992) have shown that the permeability of rock salt could be enhanced by several orders of magnitude in the cataclastic regime. In an effort to characterize permeability changes in a clay rock subjected to triaxial compressions, Zhang (2016) could highlight the effects of crack connectivity on the stress-strain-permeability behavior with post-failure permeabilities up to 5 or 6 orders of magnitude larger than the initial value. Similarly, Selvadurai and Glowacki (2017) observed permeability increase of $\sim 4$ orders of magnitude in a limestone that experienced failure caused by deviatoric loadings. However, as observed by Frash etal. (2016), permeability variation due to stress-induced 
fractures is highly dependent on the stress level at which they are generated and it should therefore be taken into account.

Several models have been proposed to complement laboratory investigations and give new insights into permeability changes caused by stress perturbations. Simpson et al. (2001, 2003) proposed analytical expressions to predict the enhancement of permeability due to stress-induced microcracking in low porosity rocks. Shao et al. (2005) proposed a constitutive model to describe anisotropic damage and permeability variation in brittle rocks under deviatoric compressive stresses through a phenomenological formalism. Jiang et al., (2010) set up a micro-mechanical based damage model for the description of anisotropic damage and permeability variation under triaxial compression able to capture the main features of mechanical behavior and permeability variations. Arson and Pereira (2013) as well as Massart and Selvadurai (2014) formulated relationships between damage and permeability with a focus on pore size change and stress-induced crack opening respectively. However, a common limitation of all these models is that they cannot reproduce the localization and coalescence of microcracks during rock failure which is known to greatly influence their hydraulic properties and could thus be a critical issue in both geoengineering and geologic contexts (Yuan and Harrison, 2006). To overcome this limitation, Lu et al. (2013) developed a 2D microcrack-based coupled continuous damage and flow model to predict the transition from diffuse microcracking to macroscopic localized fracturing together with the evolution of fluid flow due to hydro-mechanical processes. Yao et al. (2015) proposed a 2D discrete numerical model to assess permeability changes in brittle rocks with multiple fractures. Using a similar discrete approach, Tan and Konietzky (2019) used a 2D model to simulate the hydromechanical coupled behaviors of Aue granite at the grain size level and could evaluate how its permeability evolved during its progressive failure. More recently, Nguyen et al. (2020) derived an approximate model of stress-induced permeability enhancement for brittle cracked porous rocks based on linear elastic fracture mechanics, but it is only applicable for elastic and pre-peak behaviors.

The present study is motivated by the need to better characterize permeability changes occurring in lowpermeable rocks as a result of the initiation, propagation and coalescence of cracks that develop during their progressive failure. For that purpose, we developed a 3D numerical model that couples a discrete element method and a pore scale finite volume method specifically designed to describe fluid flow in cracked and fractured porous media. In this work, we put emphasis on stress-induced permeability changes occurring in a low permeable rock subjected to triaxial loadings. As such, our interest concerns short-time stress-induced permeability changes caused by fracture processes. Hence we do not consider potential thermo- or chemomechanical effects which are known to influence permeability in rocks as a result of fluid-rock interactions (see for instance Moore et al. 1994 or Guéguen et al. 1996).

The paper is organized as follows. In Sect. 2, the numerical scheme is elaborated, including the description of 
the bonded particle model used to simulate the solid phase as well as of the pore network model used to simulate fluid flow within its pore space. The capability of the proposed approach to describe fluid flow in cracked and fractured rocks is demonstrated based upon comparisons with analytical solutions from the literature. In Sect. 3 , the model is applied to simulate permeability changes in a clay rock submitted to triaxial compression tests. The stress-strain-permeability behavior is discussed based on microstructural analyses. Finally, two analytical exponential relationships between post-failure permeability and residual mean stress are proposed based on simulations conducted under several confining pressures.

\section{Methodology}

In this study, the numerical simulations are based on a method that combines a bonded particle model (BPM) and a pore-scale finite volume method (PFVM). The BPM is utilized to simulate the mechanical behavior of the rock material (Scholtès and Donzé 2013; Dinç and Scholtès 2018), while the PFVM enables to simulate fluid flow within the pore space (Chareyre et al. 2011). This coupled approach has been used in a previous study to investigate the propagation of hydraulically driven fractures in low permeability rocks (Papachristos et al. 2017).

\subsection{Bonded particle model}

The rock material is treated as a dense assembly of rigid particles interacting one with another through specific force-displacement laws. Here, following the approach proposed by Scholtès and Donzé (2013), we enable near neighbor interactions between non-strictly contacting particles so that interparticle bonds are created between pairs of particles when the following condition is fulfilled:

$$
D_{a b}^{0} \leq \gamma_{i n t}\left(R_{a}+R_{b}\right)
$$

with $R_{a}$ and $R_{b}$ the radii of the interacting particles, $D_{a b}^{0}$ the initial distance between their centroids, and $\gamma_{\text {int }}$ the interaction range coefficient, $\gamma_{i n t} \geq 1$ (Fig. 1a). This feature enables to reproduce the behavior of brittle rock like materials (see Scholtès and Donzé (2013) for details and explanations). The interparticle forces are decomposed into a normal and a tangential component (Fig. 1b).

The normal force $F_{n}$, is computed as

$$
F_{n}=k_{n} \Delta D
$$

with $\Delta D=D_{a b}-D_{a b}^{0}$ the normal relative displacement, and $k_{n}$ the normal stiffness calculated as

$$
k_{n}=2 E_{e q} \frac{R_{a} R_{b}}{\left(R_{a}+R_{b}\right)}
$$

where $E_{e q}$ is the local equivalent Young modulus. The normal force is computed with the same stiffness in compression and in tension. Unlike for compression where the interparticle force can increase infinitely, a maximum admissible tensile force $F_{n, \max }$ is defined: 


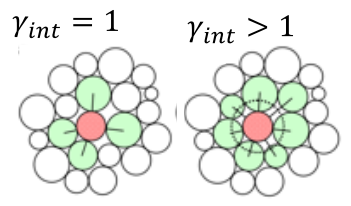

(a)

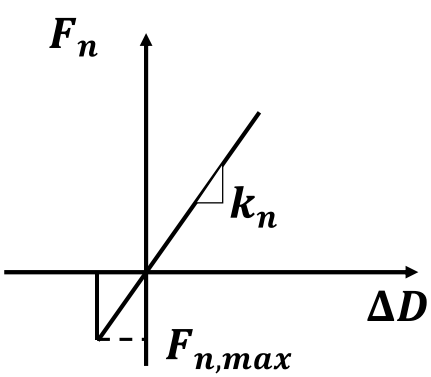

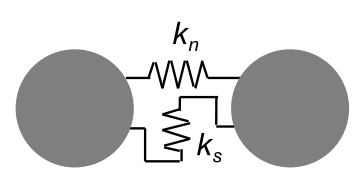

(b)

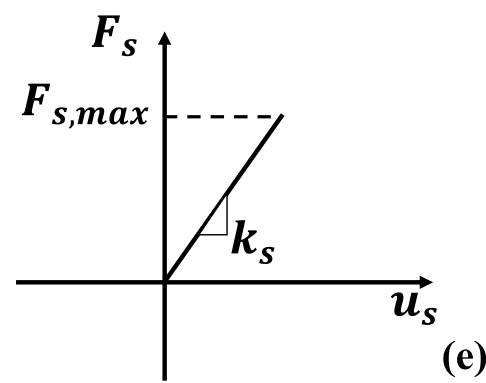

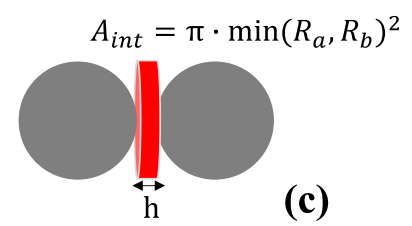

(c)

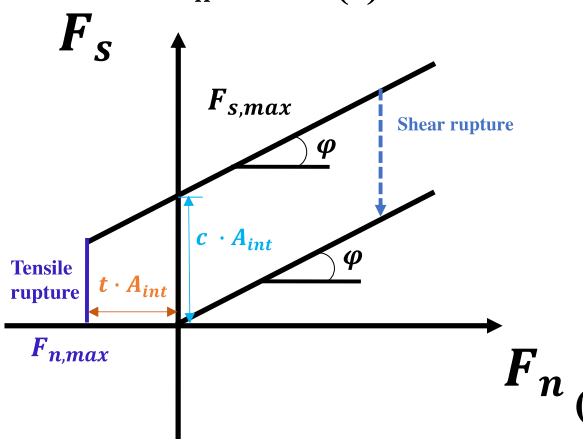

Fig. 1 (a) Effect of the interaction range $\gamma_{i n t}$ on the contact fabric, modified from Scholtès and Donzé (2013). (b) Interparticle contact model with the normal and the shear stiffness. (c) Microcrack between two formerly bonded particles characterized by its aperture $h$ and its interacting surface $A_{\text {int }}$, (d) Normal and (e) shear interaction laws, (f) Interparticle rupture criterion, modified from Scholtès and Donzé (2013).

$$
F_{n, \max }=t \cdot A_{\text {int }}
$$

with $t$ the tensile strength of the interparticle bond, and $A_{\text {int }}=\pi \cdot\left(\min \left(R_{a}, R_{b}\right)\right)^{2}$ the interacting surface area between a and b (Fig. 1c-d). When $F_{n}$ reaches its maximum value, tensile rupture occurs and a mode I crack is generated at the contact location (Fig. 1c and 1f).

The shear force $F_{S}$ is calculated in the incremental form,

$$
F_{s}=\left\{F_{s}\right\}_{\text {updated }}+k_{s} \Delta u_{s}
$$

with $\Delta u_{s}$ the relative incremental tangential displacement and $k_{s}$ the shear stiffness, which is related to the normal stiffness via the stiffness ratio $\delta$ such as:

$$
k_{s}=\delta k_{n}
$$

The maximum admissible shear force $F_{s, \max }$ is defined according to a Mohr-Coulomb criterion such as

$$
F_{s, \max }=F_{n} \tan \varphi+c A_{\text {int }}
$$

where $c$ is the local cohesion, and $\varphi$ is the local friction angle (Fig. 1e). When $F_{S}$ reaches its maximum value, shear rupture occurs and a mode II crack is generated at the contact location (Fig. 1c and 1f). The surfaces of both mode I and mode II induced cracks are equal to $A_{\text {int }}$.

The numerical scheme is based on an iterative procedure, similar to the discrete element method (DEM) scheme proposed by Cundall and Strack (1979). During each computational cycle, the positions of the particles and their contact geometry are first determined. Then the force-displacement laws are applied to calculate the forces exerted on each particle. An explicit finite difference scheme is then used to integrate Newton's second law of motion in order to update the positions of the particles. In addition, a non-viscous Cundall's type damping is used to ease the convergence of the simulated system towards quasi-static equilibrium (Duriez et al. 2016). 


\title{
2.2 Pore scale finite volume model
}

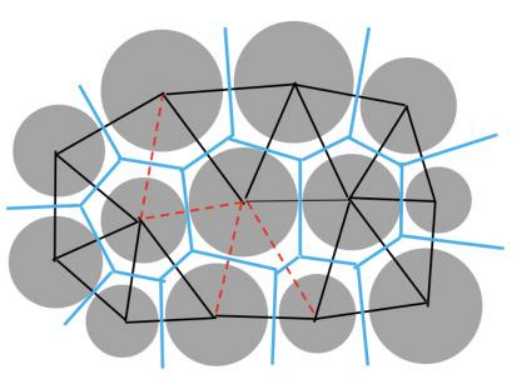

\author{
Regular triangulation \\ /Intact bond \\ - Regular Voronoi Tesselation \\ ----- Broken bond
}

(a)

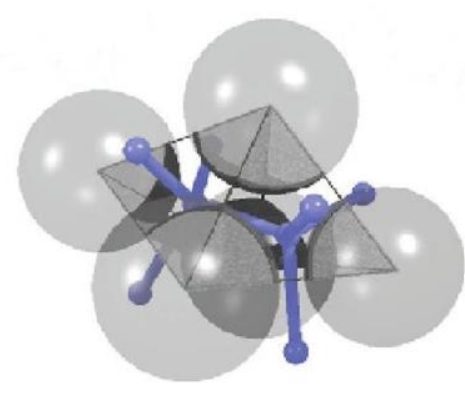

(b)
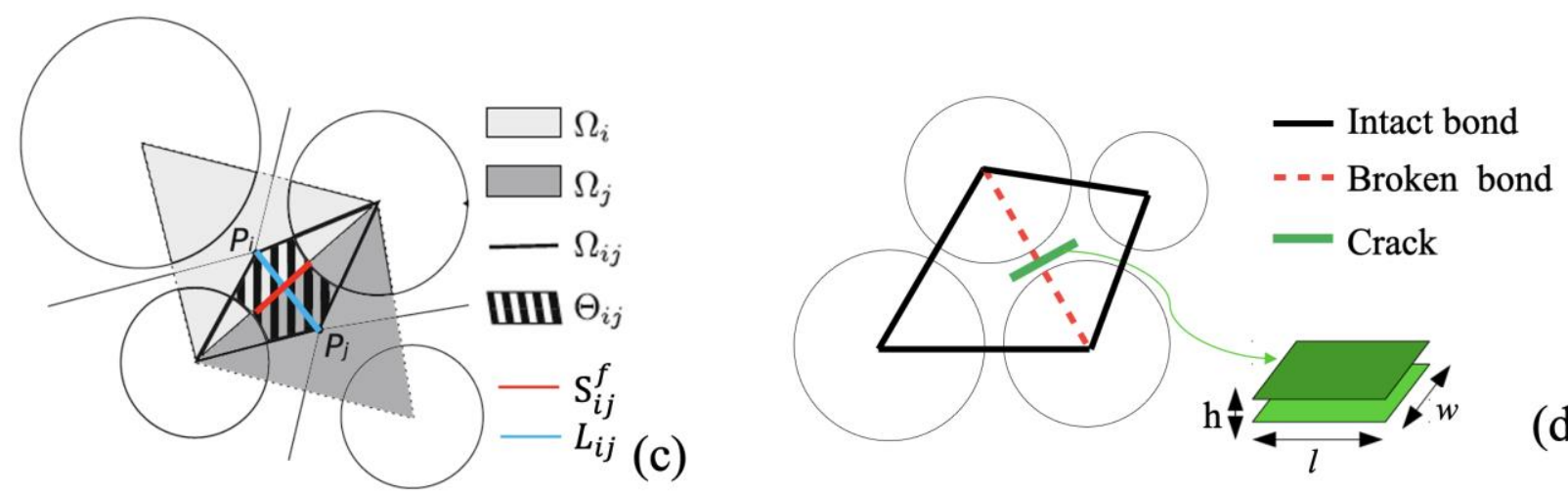

Fig. 2 (a) 2D view of a particle assembly containing both intact and broken bonds and representation of the superimposed pore network. (b) 3D view of the pore network constructed from the triangulation of the particles' centers. The Voronoï graph representing the connectivity between the pores is also shown (in blue). (c) Interpore geometry in the intact rock matrix. $\Omega_{i}$ : domain defined by tetrahedron $i, \Omega_{i j}$ : union of tetrahedra $\mathrm{i}$ and $\mathrm{j}, \Theta_{i j}$ : part of $\Omega_{i j}$ occupied by the fluid phase, $P_{i}$ : Voronoi dual (weighted center) of tetrahedron $i, S_{i j}^{f}$ : exchange surface between pores $\mathrm{i}$ and $\mathrm{j}, L_{i j}$ : length of the interpore throat $i j$. (d) Modification of the interpore connectivity in the presence of a crack (broken bond). The width of the parallel plate model $w$ is supposed equal to its length $l, h$ is the hydraulic aperture of the crack, related to the normal displacement between the debonded particles.

Chareyre et al. (2011) proposed a pore-scale model for effectively upscaling incompressible viscous flow in granular assemblies. The pore network is defined through a regular Delaunay triangulation of the particles' centers (Fig. 2a). Inter-pore fluid flow is computed thanks to a discretization of the continuity equation which can be written as:

$$
\dot{V}_{l}^{f}=-\sum_{j=1}^{4} q_{i j}=-\sum_{j=1}^{4} k_{i j}\left(p_{i}-p_{j}\right)
$$

where $V_{i}^{f}$ is the fluid volume contained in pore $i, q_{i j}$ is the fluid flux between pore $i$ and $j, k_{i j}$ is the local conductance linking pore $i$ to its neighbor $j$, and $p_{i}$ and $p_{j}$ are the fluid pressures in pores $i$ and $j$ respectively.

The model used here is based on the numerical scheme proposed in Papachristos et al. (2017) which enables to describe fluid flow in double permeability media by assigning different interpore conductances $k_{i j}$ depending on the state of the related interparticle bonds (either intact/cohesive or cracked/non cohesive as illustrated in Fig. 2). If the medium is intact (no cracks), 


$$
k_{i j}=\alpha \frac{S_{i j}^{f}\left(R_{i j}^{h}\right)^{2}}{\mu L_{i j}}
$$

where $\alpha$ is a scaling factor that can be used to match a pre-defined macroscopic permeability, $S_{i j}^{f}$ is the porethroat's cross-sectional area, $R_{i j}^{h}$ is the hydraulic radius, and $L_{i j}$ is the length of the pore throat. These last three parameters are related to the geometry of the pore network (Fig. 2b-c). Their detailed calculations can be found in Chareyre et al. (2011). The macroscopic permeability of an intact assembly thus depends both on the microstructure of the medium (e.g. the size distribution of the particles and the porosity of the assembly) and on the scaling parameter $\alpha$.

On the other hand, if an interparticle bond breaks, a crack is generated and the corresponding edge of the triangulated domain is assigned a dedicated "cracked" state. The flow through the facet associated to the cracked edges is then governed by the parallel plate model based on the cubic law (Zimmerman and Bodvarsson 1994; Zhang et al. 2006). The local interpore conductivity $k_{i j}$ is thus computed as a function of the crack's aperture $h$ which is updated at each time step of the simulation such as (Fig. 2d):

$$
k_{i j}=\frac{h^{3}}{12 \mu} \text { with }\left\{\begin{array}{l}
h=\Delta D \text { if } h>h_{r} \\
h=h_{r} \text { if } h \leq h_{r}
\end{array}\right.
$$

where $\mu$ is the dynamic viscosity of the fluid, $h$ is the local hydraulic aperture related to the normal displacement between the debonded particles. Here, we make the assumption that the width of the crack is equal to its length $L_{i j}$ to ensure consistency between Eq. (10) and the actual cubic law. A residual aperture $h_{r}$ is introduced to account for the effect of the fracture roughness or the presence of infilling material between the fracture's lips (Papachristos et al. 2017). $h_{r}$ can be predefined or set to 0 depending if the cracks are preexistent or induced.

Another thing to mention is that in the current study, the coupling between the BPM and the PFVM is one way only: the flow scheme is run periodically to simulate fluid flow within the simulated medium without inducing any forces to the solid phase. This strategy has been specifically chosen to study permeability changes resulting from external stress changes without any fluid pressure induced deformation.

One has to note that we measure the effective permeability of our numerical samples based on Darcy's law by imposing a uniaxial flow with a pressure difference $\Delta p$ across its length:

$$
k=\frac{Q \mu L}{A \Delta p}
$$

with $Q$ the volumetric flow rate at equilibrium, $A$ the cross-sectional area of the sample and $L$ the length of the sample. 
In this study, the numerical rock samples are supposed to be fully saturated with a single-phase fluid (water) and the tests performed under perfectly drained conditions. The fluid pressure difference applied for the permeability tests is equal to $1 \mathrm{~Pa}$ and the effects of pressure and temperature on the fluid properties are not considered. The fluid is assumed incompressible and its density kept constant and equal to $1000 \mathrm{~kg} / \mathrm{m}^{3}$.

\subsection{Verification of the numerical scheme}

In this section, we assess the model relevance for computing the permeability of cracked and fractured porous rocks based on two verification examples. The first example concerns the permeability tensor of a porous medium including a single fracture. The second example concerns the permeability tensor of a homogeneously cracked medium. The fractures in these simulations are simulated as pre-existing cracks with a pre-defined constant residual aperture $h_{r}$. The simulations are based on hydraulic loadings only, the medium is considered non-deformable and no mechanical loadings are applied.

\subsubsection{Permeability tensor of a porous medium with a single fracture}

The hydraulic permeability of fractured rock masses is generally anisotropic, the heterogeneity of the permeability tensor being affected by the amount of fractures and by their orientations (Ababou et al. 2011, Rong et al. 2013). Lough et al. (1998) proposed an analytical solution defining the permeability of a porous rock sample containing a single fracture as a function of the fracture orientation (Fig. 3a). In their model, the fracture is vertical ( $\mathrm{Z}$ direction), it intersects with the top and bottom surfaces of the sample while it does not cross the sample in the horizontal direction. The ratio of the fracture horizontal length to the sample length is 0.6. The fracture is centered in the sample and is oriented at an angle of $\theta$ degrees to the $\mathrm{X}$ direction. The ratio of the fracture permeability to the rock matrix permeability is set at $2.0 \times 10^{6}$. In this configuration, according to Lough et al. (1998), the effective rock permeability tensor depends on the fracture orientation $\theta$ as follows:

$$
\left\{\begin{array}{c}
k x(\theta)=k x\left(0^{\circ}\right) \cos ^{2}(\theta)+k y\left(0^{\circ}\right) \sin ^{2}(\theta) \\
k y(\theta)=k x\left(0^{\circ}\right) \sin ^{2}(\theta)+k y\left(0^{\circ}\right) \cos ^{2}(\theta) \\
k z(\theta)=k z\left(0^{\circ}\right)
\end{array}\right\}
$$

with $k x, k y$ and $k z$ the permeabilities in the $\mathrm{X}, \mathrm{Y}$ and $\mathrm{Z}$ directions, respectively.

Before verifying if our numerical model can reproduce the solution provided by Eq. (12), its hydraulic parameters should be calibrated to ensure the ratio of the fracture permeability to the rock matrix permeability is equal to $2.0 \times 10^{6}$ as assumed by Lough et al. (1998). Since Eq. (12) is suitable in general and the permeability of the intact rock matrix is not specified in Lough et al. (1998), we set the global permeability of the intact rock matrix equal to $10^{-20} \mathrm{~m}^{2}$, a reasonable value for an intact clay rock (Zhang, 2016). Thus, the permeability of the fracture should be set to $2 \times 10^{-14} \mathrm{~m}^{2}$. 

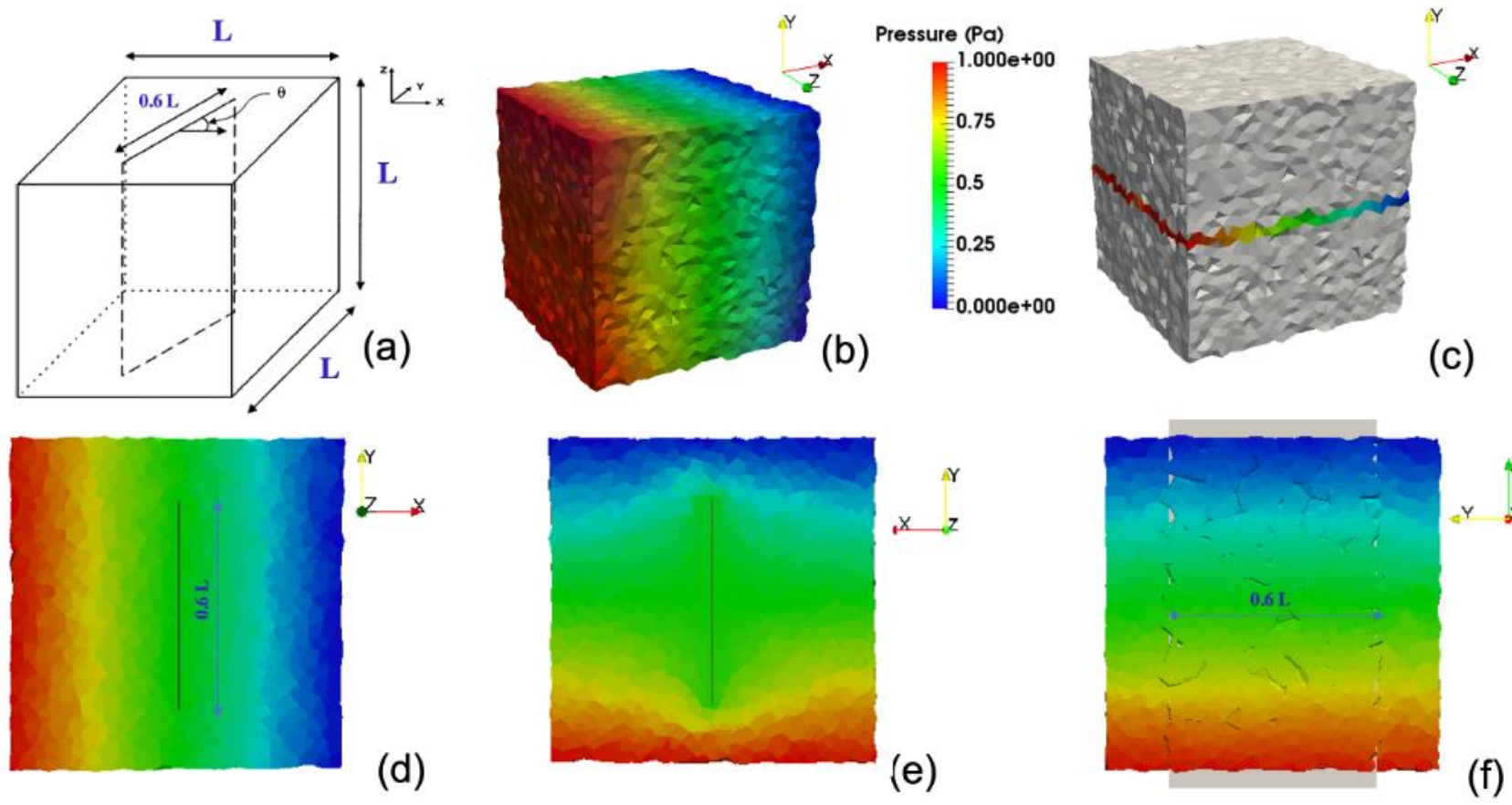

Fig. 3 (a) Geometry of the fractured sample containing a pre-existing fracture orientated at $\boldsymbol{\theta}^{\circ}$ to the $\mathrm{X}$ direction, modified from Lough et al. (1998), (b) Calibration of the matrix permeability factor $\boldsymbol{\alpha}$ : distribution of fluid pressure within the intact rock sample subjected to a $1 \mathrm{~Pa}$ pressure difference along the $\mathrm{X}$ direction (a no-flow condition is applied on the $\mathrm{Y}$ and $\mathrm{Z}$ boundaries). (c) Calibration of the cracks residual aperture $\boldsymbol{h}_{\boldsymbol{r}}$ : distribution of fluid pressure within a persistent fracture that crosses the sample subjected to a 1 Pa pressure difference along the $\mathrm{X}$ direction (a no-flow condition is applied on the $\mathrm{Y}$ and $\mathrm{Z}$ boundaries). Fluid flow is only allowed in the fracture. (d-f) Permeability test performed on the fractured porous sample: mid-length cross-sections showing the distribution of fluid pressure within the sample for a fracture oriented at $90^{\circ}$ to the $\mathrm{X}$ direction for flow in the (d) X direction, (e) $\mathrm{Y}$ direction and (f) $\mathrm{Z}$ direction. In (d)-(f), the fluid is allowed to flow through both the rock matrix and the fracture is represented as a grey plane.

Table 1 Hydraulic parameters used in the model to reproduce the analytical results of Lough et al. (1988)

\begin{tabular}{ll}
\hline Parameters & Values \\
\hline Dimension of the sample $[\mathrm{m} \times \mathrm{m} \times \mathrm{m}]$ & $0.38 \times 0.38 \times 0.38$ \\
Fracture length/Sample length & 0.6 \\
Number of particles & 20,000 \\
Calibrated permeability factor $\alpha$ & $5.0 \times 10^{-14}$ \\
Calibrated fracture aperture $h_{r}[\mathrm{~m}]$ & $5.71 \times 10^{-7}$ \\
Permeability of the intact rock $\left[\mathrm{m}^{2}\right]$ & $1 \times 10^{-20}$ \\
Permeability of the fracture $\left[\mathrm{m}^{2}\right]$ & $2 \times 10^{-14}$ \\
\hline
\end{tabular}

Two permeability tests were conducted to calibrate the permeability of the intact rock matrix and of the fracture respectively (Papachristos et al. 2017). Firstly, we built up a cubic sample with a length of $0.38 \mathrm{~m}$, composed of 20,000 particles whose radii are uniformly distributed between $4.8 \mathrm{~mm}$ to $9.6 \mathrm{~mm}$. Given a pre-defined particle packing, i.e. a pre-defined pore network geometry, the parameter $\alpha$ presented in Eq. (9) is the only parameter that needs to be scaled to match the targeted macroscopic permeability. Based on Eq. (11), we found that setting $\alpha=5.0 \times 10^{-14}$ ensures the global permeability of the intact sample $k_{m}=1.0 \times 10^{-20} \mathrm{~m}^{2}$ when simulating flow in the $\mathrm{X}$ direction (Fig. 3b). Using the same value, the global permeability in $\mathrm{Y}$ and $\mathrm{Z}$ directions are $1.0 \times 10^{-20} \mathrm{~m}^{2}$ and $1.0 \times 10^{-20} \mathrm{~m}^{2}$ respectively, indicating that the sample is homogeneous and isotropic. Secondly, we introduced a persistent fracture in the sample and simulated fluid flow within the 
fracture only by blocking flow in the matrix pores, i.e., setting $\alpha=0$ in the pores not connected to the fracture (Fig. 3c). The fracture permeability $k_{f}$ is directly related to the local hydraulic aperture $h_{r}$ defined in our model (Eq. (10)). We found that setting $h_{r}=5.71 \times 10^{-7} \mathrm{~m}$ resulted in a fracture permeability equal to $2.0 \times 10^{-14} \mathrm{~m}^{2}, 2.0 \times 10^{6}$ times the permeability of the intact rock matrix, as expected for reproducing the configuration proposed by Lough et al. (1998). All the related parameters used in this particular model are shown in Table 1.

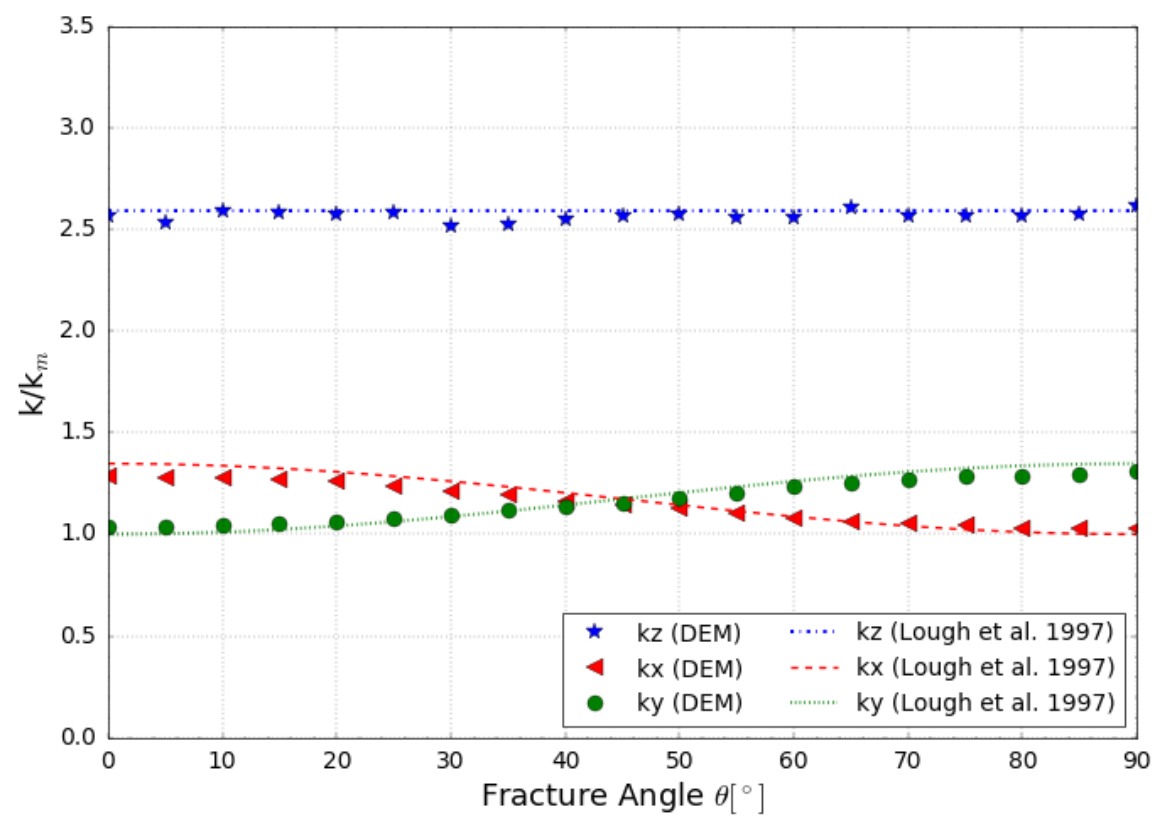

Fig. 4 Evolution of the permeability tensor components of the fractured porous sample presented in Fig. 3 as functions of the fracture orientation $\theta$ : comparison between the analytical solution by Lough et al. (1998) and the predictions of the proposed model.

Finally, keeping the calibrated values of $\alpha$ and $h_{r}$, we computed the permeability of the fractured sample defined by Lough et al. (1998) considering a $0.228 \mathrm{~m}$ length fracture embedded in the $0.38 \mathrm{~m}$ length cubic sample. The fluid is allowed to flow through both the rock matrix and the fracture. We measured permeability in all three directions $\mathrm{X}, \mathrm{Y}$ and $\mathrm{Z}$ for $\theta$ ranging from $0^{\circ}$ to $90^{\circ}$ (Fig. 3e-f). The comparison between the numerical predictions and the analytical solution is shown in Fig. 4. Overall, the model results are in very good agreement with the analytical solution. The permeability in the $\mathrm{Z}$ direction $k z$ is generally larger than $k x$ and $k y$, due to the fact that the fracture intersects the boundary surfaces of the sample in the $\mathrm{Z}$ direction, which makes the flow rate in $\mathrm{Z}$ direction much larger than in the $\mathrm{X}$ or $\mathrm{Y}$ directions. The permeability $k x$ decreases as $\theta$ increases from $0^{\circ}$ to $90^{\circ}$ because the flow rate is larger when the fracture is parallel to the flow direction $\left(\theta=0^{\circ}\right)$ compared to the case when the fracture is perpendicular to the flow direction $\left(\theta=90^{\circ}\right)$. The global permeability in the latter configuration is mainly controlled by the matrix permeability. For the same reason, the variation of $k y$ as a function of $\theta$ follows the opposite trend of $k x$. For $\theta$ equals $45^{\circ}, k x$ and $k y$ are identical. 


\subsubsection{Permeability of a cracked porous medium}

The permeability of damaged media depends on the amount of cracks they contain. Several studies have found that power-law relationships exist between permeability and fracture density (Jafari and Babadagli., 2013; Chen et al. 2018; Hyman et al. 2018). Particularly, Hyman et al. (2018) proposed that the effective permeability $k_{e}$ of cracked media can be expressed by two distinct power-laws, one for low fracture densities (Regime 1) and another for high fracture densities (Regime 2). The fracture density $\chi$ in Hyman et al. (2018) was defined as the ratio of the volume of fractures to the total volume of the domain. For instance, the two power-law relationships proposed by Hyman et al. (2018) based on their numerical model are $k_{e}=C_{1} \chi^{0.78}$ (Regime 1) and $k_{e}=C_{2} \chi^{2.50}$ (regime 2), for the case where the ratio of fracture permeability $k_{f}$ to matrix permeability $k_{m}$ equals 6 . The objective here is to assess our model with respect to the power-law relationships proposed by Hyman et al. (2018).

In order to study the influence of crack density on the permeability of cracked media, we built up a sample of dimensions $70 \mathrm{~mm} \times 140 \mathrm{~mm} \times 70 \mathrm{~mm}$ containing 20,000 particles whose radii were uniformly distributed between $1.2 \mathrm{~mm}$ and $2.2 \mathrm{~mm}$. As cracks are usually characterized with preferred orientations in clay rocks such as shale, we introduced homogeneously distributed cracks sharing the same reference orientation $\beta$ within our numerical medium. The crack aperture $h_{r}$ is predefined as $h_{r}=5 \times 10^{-7} \mathrm{~m}$, to make $\log _{10}\left(\frac{k_{f}}{k_{m}}\right)=6.22$, which is close to the value of 6.0 used in Hyman et al. (2018). $\beta$ and $h_{r}$ are kept identical for all cracks contained within the simulated medium.

The number of cracks introduced within the medium was controlled by an angle range $\Delta \beta$. The bonds that were orientated initially within $(\beta \pm \Delta \beta)$ were replaced by cracks reorientated according to $\beta$, following the method proposed in Dinç and Scholtès (2018). The crack intensity $P_{32}$ and the crack volume $P_{33}$ are introduced to quantify the crack density in the numerical samples:

$$
P_{32}=\frac{A_{\text {fracture }}}{V_{\text {total }}}\left[\mathrm{m}^{-1}\right], \quad P_{33}=\frac{V_{\text {fracture }}}{V_{\text {total }}}[-]
$$

$P_{32}$ is defined as the ratio of the total surface area of the cracks $\left(A_{\text {fracture }}=\sum A_{\text {int }}\right)$ to the total volume of the sample $\left(V_{\text {total }}\right), P_{33}$ is defined as the ratio of the total volume of the cracks $\left(V_{\text {fracture }}\right)$ to the total volume of the sample. As the crack hydraulic aperture $h_{r}$ is identical for all cracks, $P_{33}=P_{32} h_{r}$.

By varying the crack orientation $\beta$ with respect to the horizontal $\mathrm{X}$ direction as well as $\Delta \beta$, different distributions of cracks were tested (Fig. 5a-b). Under smaller values of $\Delta \beta$, few cracks are generated and they are isolated from each other. As $\Delta \beta$ increases, the amount of cracks increases and their degree of connectivity gets higher. It can be seen from Fig. 6 that, for given values of $\beta$ and $h_{r}, P_{33}$ as well as the ratio of predefined cracks to the total number of contacts increase with the increase of $\Delta \beta$ from $0^{\circ}$ to $90^{\circ}$. 

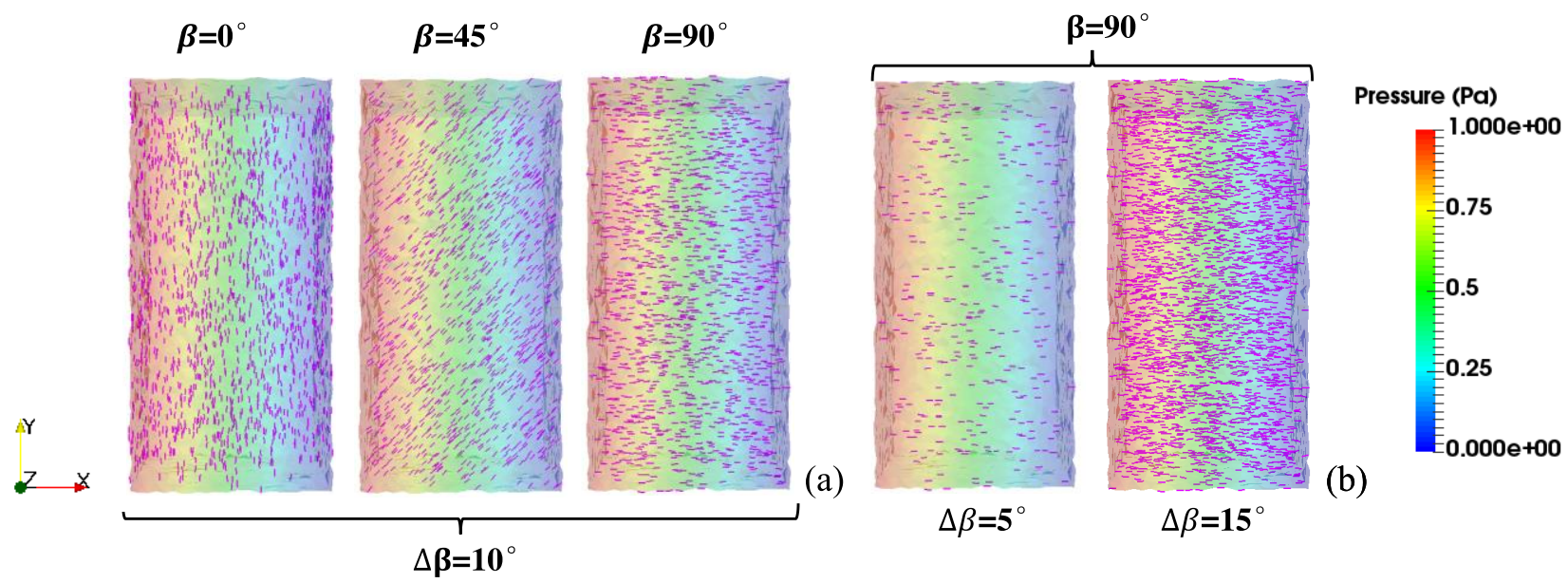

Fig. 5 Distributions of fluid pressure within homogeneously cracked samples during permeability test simulations (flow induced by a 1 $\mathrm{Pa}$ pressure difference along the $\mathrm{X}$ direction). The number of pre-existing cracks is defined by varying $\Delta \boldsymbol{\beta}$. Their orientation $\boldsymbol{\beta}$ is imposed. Each short magenta line represents a crack. (a) The three images from left to right correspond to $\boldsymbol{\beta}=0^{\circ}, \boldsymbol{\beta}=45^{\circ}$ and $\boldsymbol{\beta}=90^{\circ}$ respectively. For all these cases $\Delta \boldsymbol{\beta}=10^{\circ}$. (b) The two images from left to right correspond to $\Delta \boldsymbol{\beta}=5^{\circ}$ and $\Delta \boldsymbol{\beta}=15^{\circ}$, respectively. For both cases $\boldsymbol{\beta}=90^{\circ}$.

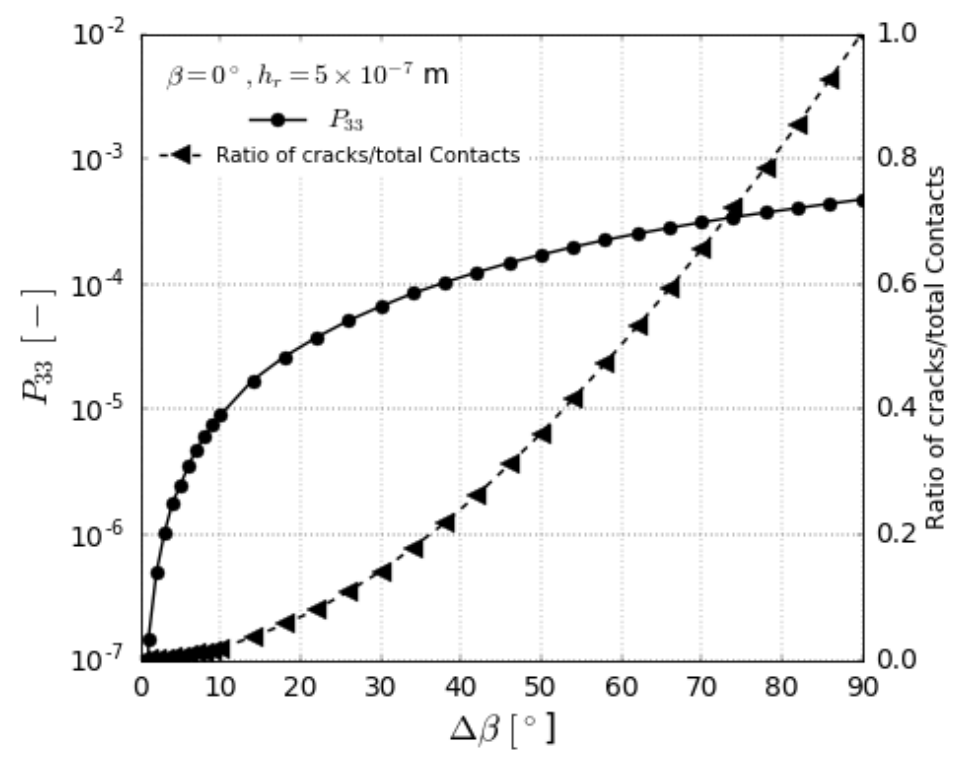

Fig. 6 Evolutions of the crack volume $P_{33}$ and of the ratio of pre-defined cracks over the total number of contacts as functions of $\Delta \beta$ for $\beta=0^{\circ}$ and $h_{r}=5 \cdot 10^{-7} \mathrm{~m}$.

The evolutions of $k x, k y$ and $k z$ as functions of $P_{33}$ for $\beta=0^{\circ}$ and $h_{r}=5 \times 10^{-7} \mathrm{~m}$ is shown in Fig. 7. The permeabilities increase as $P_{33}$ increases. As in Hyman et al. (2018), two power-laws exist, one for smaller values of $P_{33}$ (Regime 1: $k=8 \times 10^{-21}\left(P_{33}\right)^{0.013}$ ), and another one for larger values of $P_{33}$ (Regime 2: $k=8 \times 10^{-4}\left(P_{33}\right)^{4.2}$ ). The transition between the two regimes corresponds to $P_{33}=8.42 \times$ $10^{-5}$. For the first regime, the permeabilities are mainly controlled by the rock matrix permeability as the cracks are not connected. The exponent value for the first power-law is 0.013 , which is much smaller than the 0.78 proposed by Hyman et al. (2018), whereas the exponent value for the second power-law is 4.2 , which is 
much larger than the 2.5 proposed by Hyman et al. (2018). These differences might be related to the fact that the fractures are randomly orientated in Hyman et al. (2018), while they have pre-defined orientations in this particular study, and also that the fractures do not follow the same size distribution in both cases.

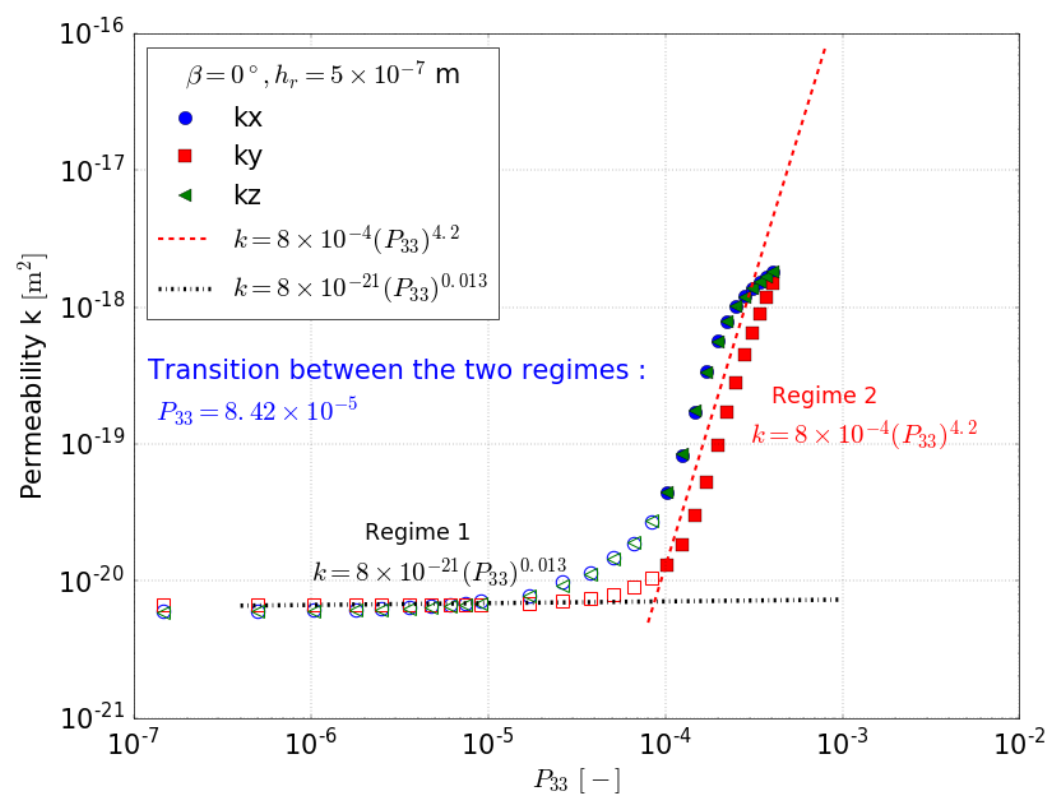

Fig. 7 Evolutions of the permeability tensor components within homogeneously cracked samples predicted by the proposed model as functions of $P_{33}$ for $\beta=0^{\circ}$ and $h_{r}=5 \times 10^{-7} \mathrm{~m}$. Permeability values associated with Regime $2\left(k=8 \times 10^{-4}\left(P_{33}\right)^{4.2}\right)$ are represented by filled markers while values associated with Regime $1\left(k=8 \times 10^{-4}\left(P_{33}\right)^{0.013}\right)$ are represented by empty markers. Colors indicate the principal direction of the flow, X: blue, Y: red, and Z: green.

The permeability in the $\mathrm{Y}$ direction $k y$ is generally smaller than $k x$ and $k z$ because for $\beta=0^{\circ}$, the cracks are perpendicular to the direction of the $\mathrm{Y}$ flow while they are parallel to the flow in both $\mathrm{X}$ and $\mathrm{Z}$ directions. For larger crack densities, the fractures are connected and the effective permeability reaches a maximum value (Fig. 7). A similar trend has been observed by Yazdi et al. (2011) who considered randomly distributed cracks. The comparison of the permeability tensors obtained by the current model and models from the literature proves the relevance of the proposed numerical scheme to compute permeability of cracked samples. We have conducted parametric studies with different values of the residual crack aperture $h_{r}\left(1 \times 10^{-6} \mathrm{~m}\right.$ and $2 \times$ $\left.10^{-6} \mathrm{~m}\right)$ and of the crack orientation $\beta\left(0^{\circ}\right.$ and $\left.90^{\circ}\right)$ and similar trends were obtained (results are provided in the Appendix C).

\section{Crack permeability behavior of a clay rock under triaxial compression}

Zhang (2016) experimentally investigated the complete pre- and post-failure stress-strain-permeability behavior of the Callovo-Oxfordian claystone by performing triaxial compression tests with periodic permeability measurements under confining stresses varying from $0.5 \mathrm{MPa}$ up to $12 \mathrm{MPa}$. Permeability was estimated by injecting Nitrogen gas at the bottom of the sample at a constant pressure of $0.2 \mathrm{MPa}$ and by measuring the outflow at the top at atmospheric pressure (0.1 MPa). 
We set up our numerical model to simulate the experiments performed by Zhang (2016). Since the gas pressure (0.2 $\mathrm{MPa}$ ) used in the experiments was small compared to the confining pressures, we considered that hydromechanical couplings were limited, in agreement with the one-way coupling scheme we used (see section 2.2). As in the experiment, we measured the effective permeability of the numerical sample periodically during triaxial compression loadings by applying a low fluid pressure difference across its length at given deformation stages. Hence, the variation of the permeability is directly related to the evolution of the sample microstructure due to boundary stress variations.

Sect. 3.1 presents the model set-up and its calibration. In Sect. 3.2, the stress-strain-permeability response obtained for a confining pressure of $1 \mathrm{MPa}$ is analyzed in relation to the evolution of the sample microstructure. Finally, in Sect. 3.3, we evaluate potential relationships between the post-peak permeability and the mean residual stress.

\subsection{Model set up and calibration}

Table 2 Parameters used to simulate the Callovo-Oxfordian claystone tested by Zhang (2016)

\begin{tabular}{ll}
\hline Parameters & Values \\
\hline Dimension of the sample $[\mathrm{mm} \times \mathrm{mm} \times \mathrm{mm}]$ & $70 \times 140 \times 70$ \\
Number of particles & 20,000 \\
Density of the sample $\left[\mathrm{kg} / \mathrm{m}^{3}\right]$ & 2530 \\
Initial porosity & 0.362 \\
Interaction range coefficient $\gamma_{i n t}$ & 1.067 \\
Permeability factor $\alpha$ & $4.3 \times 10^{-13}$ \\
Damping coefficient & 0.4 \\
Equivalent Young's modulus $E_{e q}[\mathrm{GPa}]$ & 10 \\
Local stiffness ratio $\delta=k_{s} / k_{n}$ & 0.1 \\
Local friction angle $\varphi\left[{ }^{\circ}\right]$ & 7 \\
Tensile strength $t[\mathrm{MPa}]$ & 12 \\
Cohesion $c[\mathrm{MPa}]$ & 12 \\
\hline
\end{tabular}

As in the experiments, the size of the numerical sample is $70 \mathrm{~mm} \times 140 \mathrm{~mm} \times 70 \mathrm{~mm}$ for the $\mathrm{X}, \mathrm{Y}$ and $\mathrm{Z}$ dimensions, respectively. The sample includes 20,000 particles whose radii are uniformly distributed between $1.2 \mathrm{~mm}$ and $2.2 \mathrm{~mm}$. A particle does not represent a unique mineral grain explicitly, but rather defines a volume of grains which represents the resolution of the numerical model. The 20,000 particles' sample was chosen here because it provides mesh objective solutions (i.e., independency of the results regarding the size of the discrete element) while limiting the computational cost. The sample is considered initially intact, without any cracks introduced a priori. Triaxial compression loadings under different confining pressures were conducted with an axial strain rate small enough to ensure the quasi-static response of the model and rate independent behaviors. 
The model parameters were calibrated to simulate the behavior of the Callovo-Oxfordian claystone tested by Zhang (2016). They are presented in Table 2.
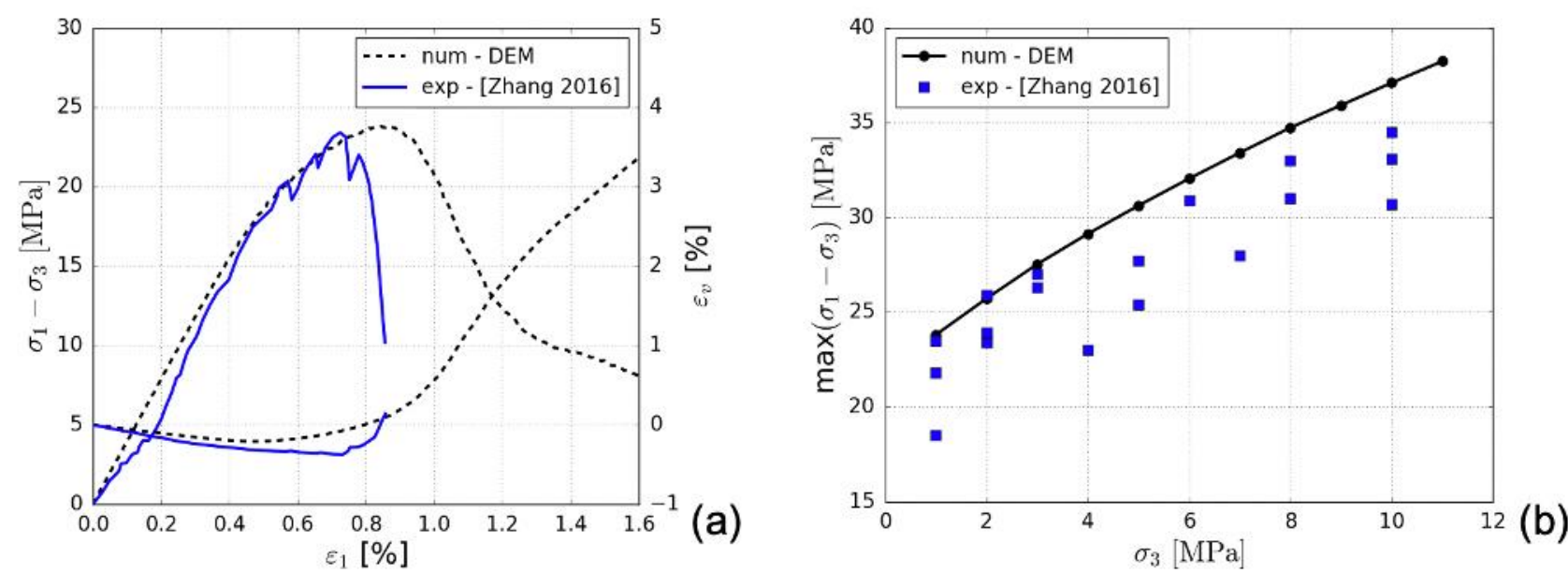

Fig. 8 (a) Stress-strain response of an argillaceous rock sample (Callovo-Oxfordian clay rock) subjected to a triaxial compression test performed under a confining pressure of $1 \mathrm{MPa}$ : comparison between the prediction of the proposed model and the experiment by Zhang (2016). (b) Comparison of experimental and numerical failure envelopes.

Given the limited number of full stress-strain curves provided in (Zhang, 2016), the comparison between experimental and numerical stress-strain relationships is firstly conducted for a triaxial loading under lateral confining pressure of $1 \mathrm{MPa}$ (Fig. 8a). For this loading case, the numerical model is able to reproduce the peak value of the differential stress, the global stress-strain slope before the peak, the strain softening as well as the dilatancy following the initial contractancy of the medium. The failure envelope obtained for triaxial loadings conducted under different confining pressures is presented in Fig. 8b. The numerical model tends to slightly overestimate the strength of the rock material. One can note that the numerical failure envelope is obtained based on the same initial sample packing and the same set of numerical parameters whereas the experimental data were obtained based on different samples and the results show large variabilities. Despite small discrepancies, the numerical model can reasonably reproduce the experimental behaviors.

\subsection{Hydraulic responses with microstructure evolutions}

We analyze here the permeability changes that occurs during the triaxial loading performed under a confining pressure of $1 \mathrm{MPa}$ in relation with the evolution of the microstructure of the medium. The permeability is periodically measured using Darcy's law by applying a $1 \mathrm{~Pa}$ fluid pressure difference in the Y direction. Because our sample does not contain any pre-existing cracks, its initial permeability is directly controlled by the equant pores of the particle assembly (Eq. (9)). The permeability factor $\alpha$ was thus previously calibrated to $4.3 \times$ $10^{-13}$ to ensure an initial permeability equals to $6.0 \times 10^{-21} \mathrm{~m}^{2}$, a value that corresponds to the permeability measured by Zhang (2016). During the loading phase, permeability changes are directly related to the volumetric deformation of the sample as well as to the stress induced cracks which, in our model, can be either tension or 
shear induced (see Sect. 2.1). Here, we made the choice to set up the residual hydraulic aperture of the induced cracks $h_{r}=0$ (Eq. (10)).

In our model, the parallel plate model can be applied to either the tensile cracks, the shear cracks or both cracks (see section 2.2 for details). Therefore, different options are available to account for the effects of stress induced cracks on the permeability. Former studies have highlighted the role of tensile cracks for the enhancement of permeability (Tan and Konietzky 2019). However, the role of shear cracks on the permeability are still not clear. Bonnelye et al. (2017) performed conventional triaxial tests on a series of samples of Tournemire shale along different orientations relative to the bedding. Extensive $\mathrm{P}$ and $\mathrm{S}$ wave elastic velocity measurements highlighted the presence of plastic mechanisms and mineral reorientation during deformation. This reorientation of minerals was also proven by scanning electron microscope images of the failure plan of the deformed samples. This plastic deformation related mineral reorientation might contribute to the decrease of the permeability. Based on the observations made by Bonnelye et al. (2017), we conducted preliminary numerical simulations in which shear cracks were not considered as permeability enhancers, applying the parallel plate model to the tension induced cracks only. The results showed a good agreement with the permeability changes observed in the experiments (see the Appendix B.1). Therefore, in the following, the calculation of $k_{i j}$ for facets containing tensile crack adopts the parallel plate model regime, while $k_{i j}$ is computed following the rule defined for the intact rock matrix for facets containing shear cracks.

In the experiment, the permeability was measured by using gas as the percolating fluid, while water is used in the numerical simulations. General discrepancies between gas and liquid permeability are discussed in the Appendix B.2. Since no information is provided in the experiments to allow the transformation of the gas permeability to the liquid permeability for quantitative comparisons, the comparisons we make here between experimental permeabilities and numerical ones are based on their overall evolutions.

The stress-strain-permeability behavior of the sample under 1 MPa confining pressure is shown in Fig. 9a where the values of the axial strain $\varepsilon_{1}$ are normalized by the axial strain corresponding to the peak stress $\varepsilon_{1}^{\text {peak }}$. The permeability measurement is conducted in the $\mathrm{Y}$ direction (axis of the sample, Fig. 9b). As in the experiment, the model is able to reproduce the rapid increase of permeability after the peak stress, which could be considered as a transition point where stress induced cracks percolate. The initial decrease of permeability predicted by the numerical model is limited compared to the one observed in the experiment. It is because the numerical sample is initially intact and does not contain pre-existing cracks which could close during the initial stage of the loading. The compaction of the model is only due to the elastic compaction of the contact network caused by small elastic particles' movements. Then, unlike in the experiment, the model predicts a small progressive increase of permeability before the peak stress is reached. This difference might be related either to differences in terms of volumetric deformation (early dilatancy in the model caused by microstructural differences, e.g., 
bedding planes in the clay rock vs. spherical grains in the model), or to the use of nitrogen gas in the experiment. Discussions can be found in the Appendix B.2 regarding this discrepancy.
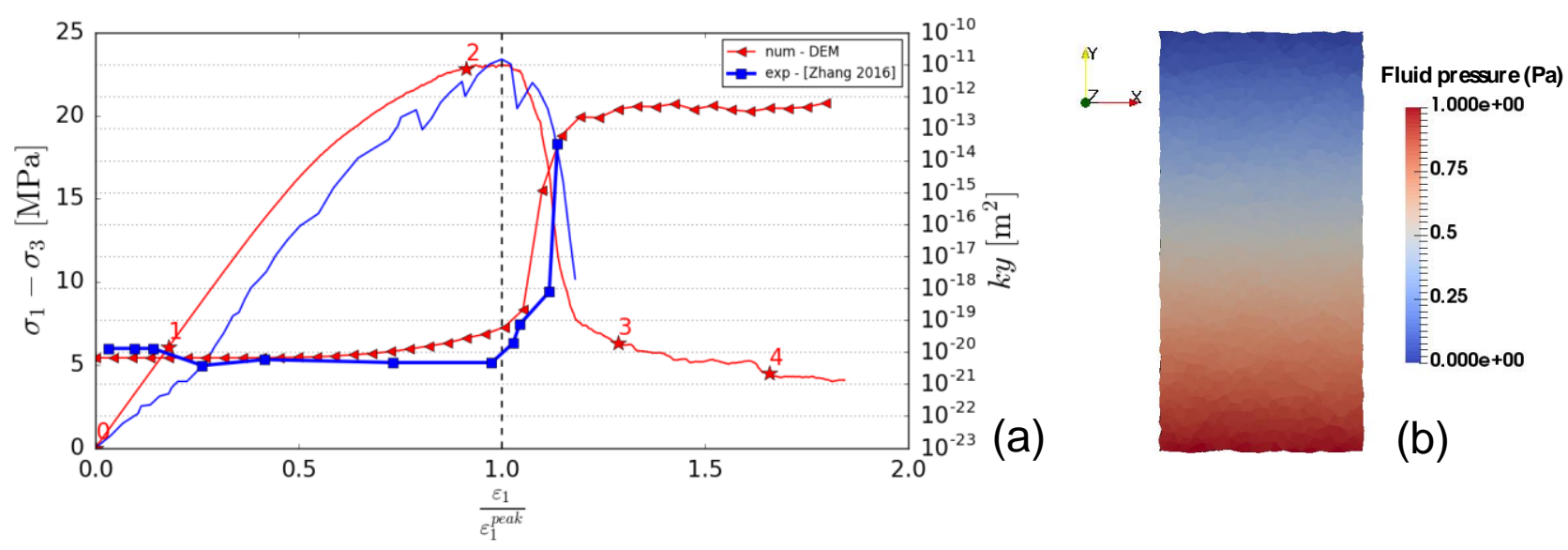

Fig. 9 (a) Stress-strain-permeability evolution of an argillaceous rock sample (Callovo-Oxfordian clayrock) subjected to a triaxial compression performed under a confining pressure of $1 \mathrm{MPa}$ : comparison between the prediction of the proposed model and the experiment by Zhang (2016). The values of the axial strain $\varepsilon_{1}$ are normalized by the axial strain at the peak stress $\varepsilon_{1}^{\text {peak }}$. (b) Distribution of fluid pressure within the numerical sample during a permeability test (this pressure map corresponds to the test performed at the initial stage of the deviatoric loading).

The stress-strain response can be segmented into four loading stages related to characteristic microstructural changes. The differential strain field, the spatial distributions of tensile cracks and shear cracks, and the pore fluid velocity field are presented in Fig. 10 for each one of these stages.

During stage $0-1$, the stress increases linearly, differential strain is limited, no microcracks are created within the sample and the global permeability remains equal to the initial one. During stage 1-2, the stress increases progressively up to the peak value and strain starts to localize along conjugated bands distributed all over the sample. A large amount of microcracks nucleate within the sample in a homogeneously distributed manner, $98 \%$ of them being tensile cracks and $2 \%$ being shear cracks. Nonetheless, the permeability does not increase significantly (less than one order of magnitude): the microcracks are not yet connected and their average aperture remains small (see the Appendix A.1). Stage 2-3 corresponds to the post-peak strain softening phase during which strain localizes in the form of a discrete shear band that crosses the sample. A large amount of microcracks are generated, $76 \%$ being tensile cracks and $24 \%$ being shear cracks. More than $50 \%$ of the cracks are localized within the shear band. The later the cracks are generated, the more likely they appear within the shear band. The permeability increases significantly as the shear band constitutes a preferential pathway for the fluid as indicated by the pore fluid velocity map: the microcracks generated within the shear band are connected and enables the fluid to percolate across its length. During stage 3-4, the differential stress continues to decrease gently down to a residual stress while all the strain is localized within the shear band where only few cracks are generated, mainly due to particle rearrangements. The microcracks generated during stage 3-4 are all located within the shear band, $77 \%$ of them being tensile cracks. The permeability does not change during this residual stage. The interconnected crack network established during the strain softening stage remains: the shear band 
localizes both the strain and the flow, similarly to a fault gouge undergoing shear. Overall, as in the experiments, the evolution of the permeability of this low permeable brittle rock is governed by the initiation, growth and coalescence of microcracks up to failure. In the post-failure regime, the stress-permeability behavior of the medium is completely governed by the shear band properties.

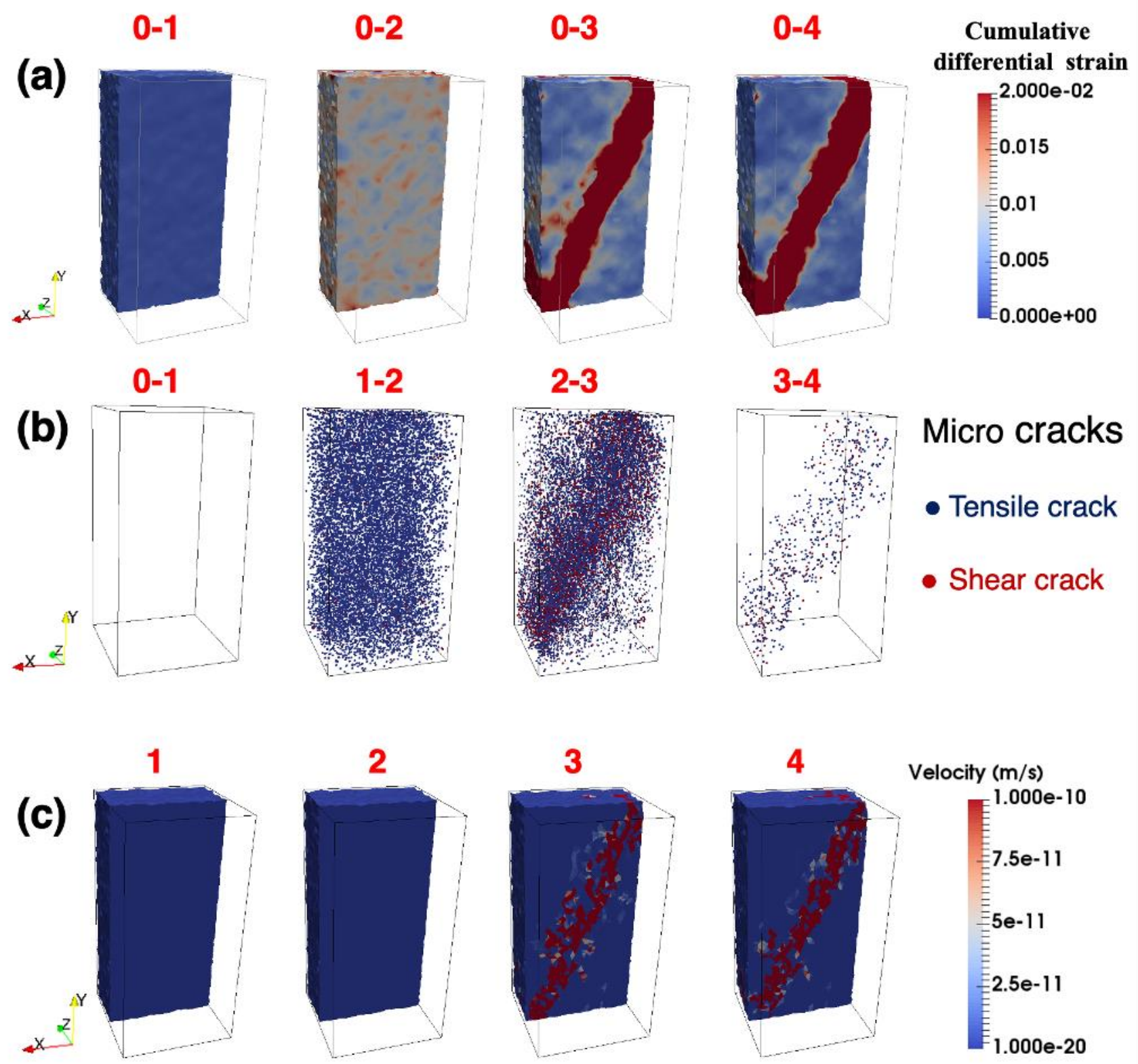

Fig. 10 Crack permeability behavior of the numerical sample subjected to a triaxial compression performed under a confining pressure of $1 \mathrm{MPa}$ : (a) cumulative differential strain fields, (b) sequential distributions of microcracks (tensile cracks are classified as type 1 (blue), shear cracks are classified as type 2 (red)) and (c) pore fluid velocity fields obtained during the permeability tests (1 Pa pressure difference applied along the vertical direction).

\subsection{Relationship between post-failure permeability and residual mean stress}

To further characterize the crack permeability behavior of low permeable rocks, we analyzed the stress-strainpermeability response of the numerical medium for triaxial loadings conducted under different confining pressures. The stress-strain-permeability responses and the cumulative differential strain for $1 \mathrm{MPa}, 10 \mathrm{MPa}$ 
and $20 \mathrm{MPa}$ are presented in Fig. 11a-c. Similar to the experiments of Zhang (2016), the peak values of differential stress increase as the confining pressure increases while the post-peak permeability decreases as the confining pressure increases. In the experiments of Zhang (2016), for confining pressures equal to 1, 5 and 10 $\mathrm{MPa}$, the final permeability values measured at failure (which corresponds to $1-2 \%$ of axial strain) are $10^{-13}$ to $10^{-14} \mathrm{~m} 2,10^{-16} \mathrm{~m} 2$ and $10^{-18}$ to $10^{-22} \mathrm{~m} 2$, respectively. In the simulations, for confining pressures equal to $1 \mathrm{MPa}, 10 \mathrm{MPa}$ and $20 \mathrm{MPa}$, the post-peak permeability measured at $5 \%$ of axial strain are $10^{-11} \mathrm{~m} 2$, $10^{-13} \mathrm{~m} 2$ and $10^{-19} \mathrm{~m} 2$. These quantitative differences between experimental and numerical post-peak permeabilities, could be due to the fact that these values are not evaluated at the same axial strain, and also because the experimental data show variabilities.
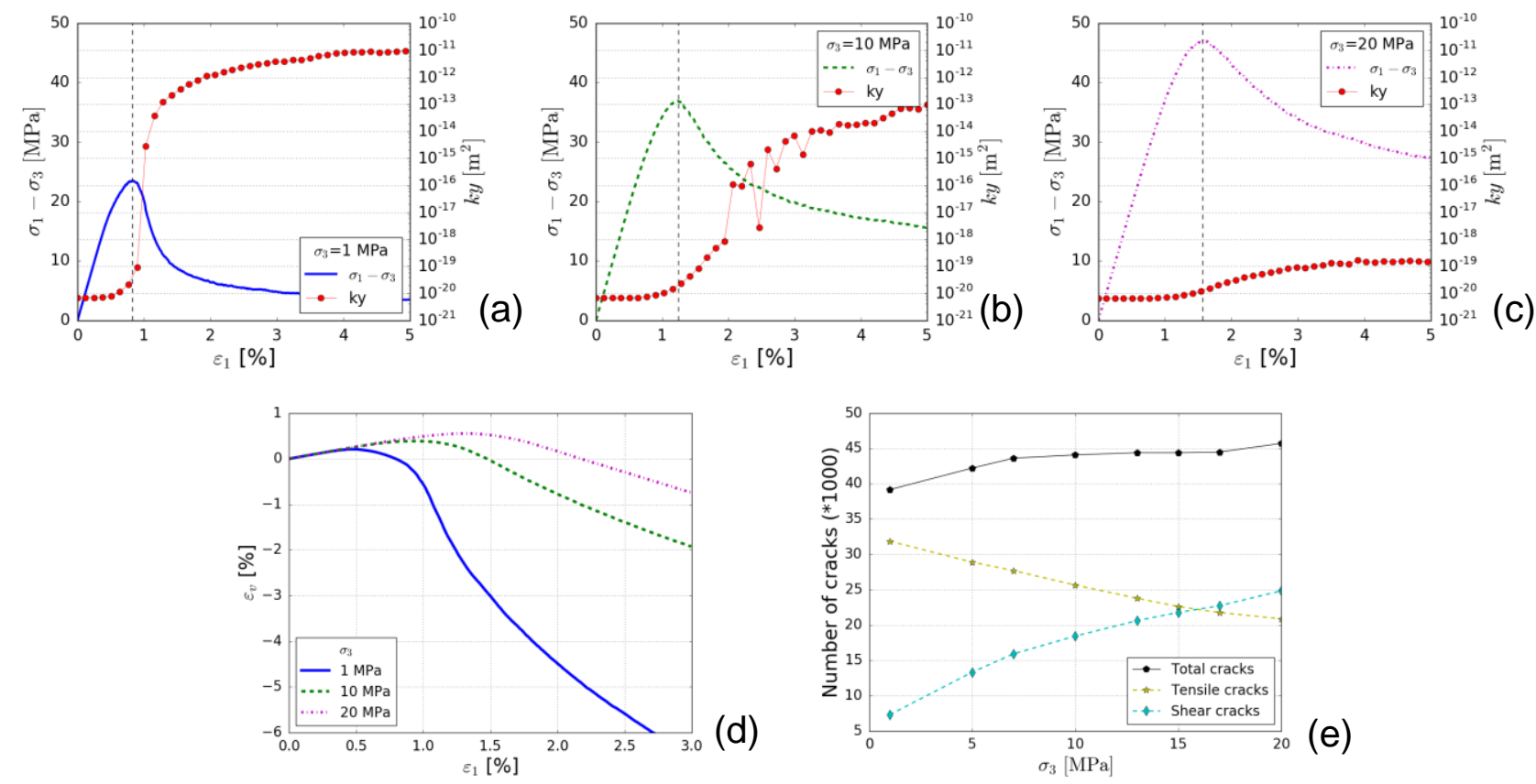

(e)

Fig. 11 Stress-strain-permeability evolutions obtained for triaxial compression test simulations performed under confining pressures respectively equal to (a) $1 \mathrm{MPa}$, (b) $10 \mathrm{MPa}$ and (c) $20 \mathrm{MPa}$. (d) Evolutions of the volumetric strain for the same triaxial compression tests. (e) Evolutions of the cumulative number of cracks generated at the end of the simulations as functions of the confining pressure.

The decrease of the post-peak permeability with the increase of the confining pressure is related to the ability (or inability) for the medium to dilate during its deformation. The larger the confining pressure, the smaller the dilatancy (Fig. 11d). Conjointly, tensile cracks tend to be inhibited when the confining pressure increases (Fig. 11e), hence limiting the appearance of preferential pathways for the fluid. As for most brittle materials, tensile cracks (Mode I) are predominant at low confining pressures, while shear cracks (mode II) become more and more significant as the confining pressure increases (Melin, 1989). Permeability enhancement is thus directly affected by the pressure dependent cracking modes experienced by the medium.

Because post-failure permeability is of interest in many geoengineering applications, the following investigation focuses on the relationship between the post-failure permeability and the residual mean stress from simulations conducted under confining pressures varying from $1 \mathrm{MPa}$ to $25 \mathrm{MPa}$. Presented in Fig. 12 are the post-failure 
permeabilities measured at the end of the simulations (5\% of axial strain). $\sigma_{e}$ is the residual mean stress also measured at $5 \%$ of axial strain, $\sigma_{e}=\left(\sigma_{1}+\sigma_{2}+\sigma_{3}\right) / 3$. Simulations were repeated on different samples for a better representativeness.

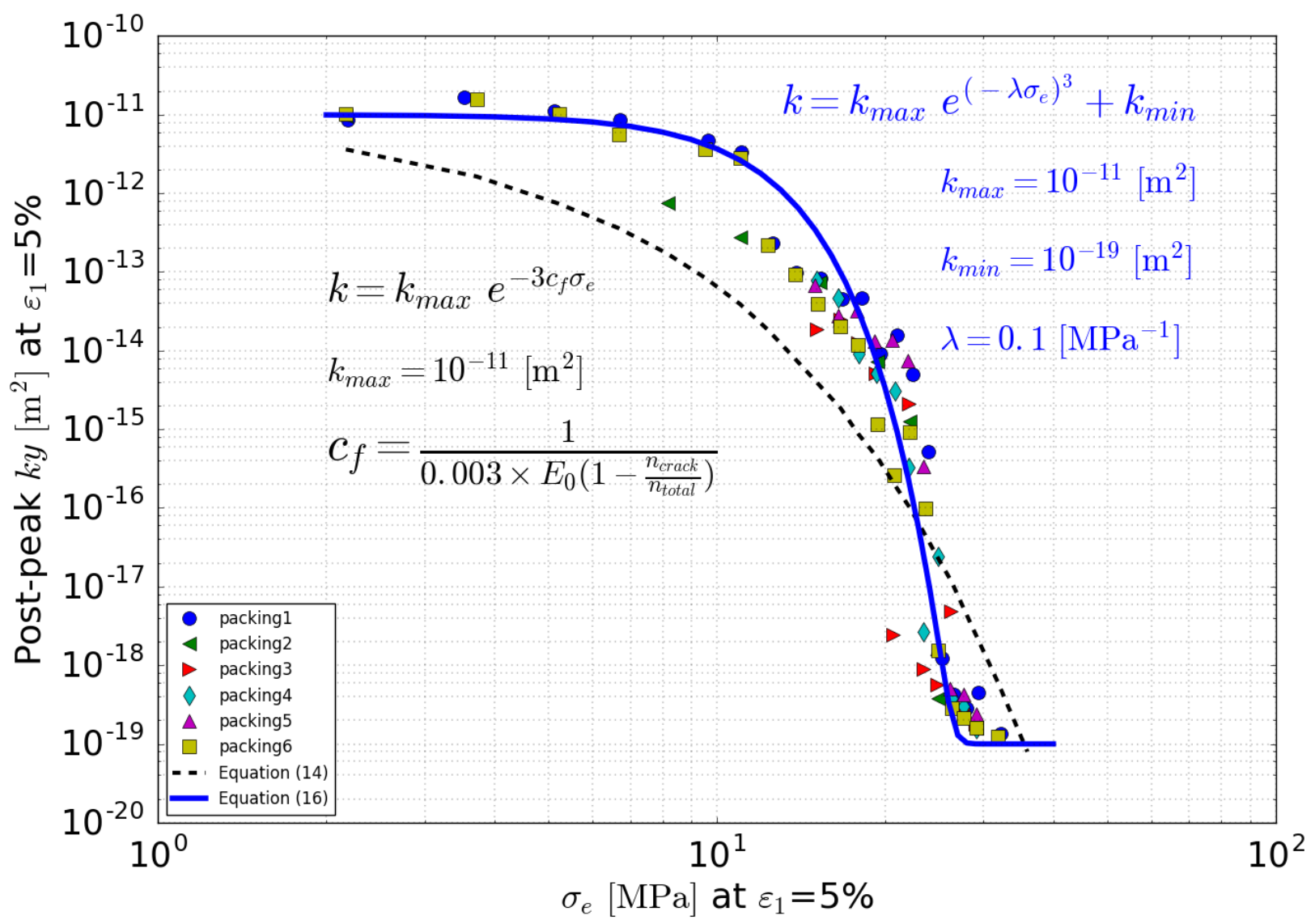

Fig. 12 Evolution of the residual (post-failure) axial permeability as a function of the residual mean stress for triaxial test simulations conducted under different confining pressures. Both the permeability and the mean stress were measured for an axial strain equal to $5 \%$.

Generally, the post-failure permeability decreases with the increase of residual mean stress. Three regimes with different declining rates can be identified. The declining rate seems to be the largest for $\sigma_{e}$ between $17 \mathrm{MPa}$ and $27 \mathrm{MPa}$ (the corresponding lateral confining pressures $\sigma_{3}$ vary from $11 \mathrm{MPa}$ to $19 \mathrm{MPa}$ ), while it is the smallest for $\sigma_{e}$ between $29 \mathrm{MPa}$ and $35 \mathrm{MPa}$ (the corresponding $\sigma_{3}$ vary from $20 \mathrm{MPa}$ to $25 \mathrm{MPa}$ ). The declining rate for $\sigma_{e}$ smaller than $16 \mathrm{MPa}$ is in between (corresponding to $\sigma_{3}$ smaller than $11 \mathrm{MPa}$ ). Similarly, different declining rates have also been reported in the literature. For instance, Ye et al. (2017) investigated the pressure-dependent permeability of shale fractures by conducting hydrostatic flow tests. They observed that the flow rate decreases with the effective confining pressure as an exponential function. In Ye et al. (2017), the declining rate is observed to be relatively smaller for confining pressures around $20 \mathrm{MPa}$, compared to those obtained for 5 and $10 \mathrm{MPa}$.

The evolutions of the numerical permeabilities can be approximated with mathematical expressions. Chen et al. (2015) established an exponential function to describe the dependance of permeability on effective stress for 
shale rocks. By modifying this exponential function, it is possible to relate the post-peak permeability to the residual mean stress:

$$
k=k_{\max } e^{-3 c_{f} \sigma_{e}}
$$

with $c_{f}$ the fracture compressibility and $k_{\max }$ the upper limit of the post-failure permeability. As the compressibility of fractures can be related to the number of fractures (Sheng et al. 2019), an assumption is made in this study to relate $c_{f}$ to a damage index, which can be related to the macroscopic Young modulus of the intact rock material $E_{0}$ as well as to the ratio of the number of cracks $n_{\text {crack }}$ to the number of initial bonds $n_{\text {total }}$.

$$
c_{f}=\frac{1}{A E_{0}(1-D)}=\frac{1}{A E_{0}\left(1-\frac{n_{\text {crack }}}{n_{\text {total }}}\right)}
$$

with $A$ a scaling parameter.

To match the declining rates obtained in the numerical simulations, the parameters present in Eq. (14) have the following values: $A=0.003, k_{\max }=1.0 \times 10^{-11} \mathrm{~m}^{2}$ and $E_{0}=4 \mathrm{GPa}$. According to Fig. 12, although the calibrated Eq. (14) can capture the general decreasing trend of the post-peak permeability, it fails to capture the three declining rates.

Because of the sharp transition of the permeability, an alternative relationship is proposed to capture the different decreasing rates of the post-peak permeability with $\sigma_{e}$ :

$$
k=k_{\max } e^{-\left(\lambda \sigma_{e}\right)^{3}}+k_{\min }
$$

with $\lambda$ a scaling parameter, $k_{\min }$ the lower limit of the post-peak permeability value obtained under the maximum confining pressure used in this study (25 MPa). The parameters of Eq. (16) are fitted to match the numerical data: $k_{\max }=1.0 \times 10^{-11} \mathrm{~m}^{2}, k_{\min }=10^{-19} \mathrm{~m}^{2}$ and $\lambda=0.1 \mathrm{MPa}^{-1}$. According to Fig. 12 , the numerical values are better fitted with Eq. (16) compared to Eq. (14), especially in terms of the three different declining rates. However, its general use for real low permeable rocks still requires further investigations, as well as a physical basis for the scaling parameter $\lambda$.

\section{Conclusion}

A discrete numerical model is proposed to investigate the crack permeability behavior of low permeable rocks. The model couples a discrete element method (DEM) for simulating the mechanical behavior of the solid phase and a pore scale finite volume method (PFVM) for simulating fluid flow within its pore space. In order to model rock like materials as dual permeability media, the inter-pore conductance is defined as a function of the mechanical state of the material which can be either intact or cracked. 
The proposed approach is first verified against permeability solutions from the literature. The model is able to predict the evolution of the permeability tensor of a fractured porous rock containing a unique pre-existing fracture as a function of the fracture orientation. The model is also able to reproduce power-law relationships relating permeability and fracture density of microcracked media, considering fracture densities either smaller or larger than a transition value.

Motivated by the need to characterize the complete stress-strain-permeability response of low-permeable rocks during their progressive failure, the proposed approach is used to simulate permeability changes occurring in a claystone sample subjected to a triaxial compression under low confining pressure (1 MPa). The model can reproduce both the pre- and post-failure behaviors observed experimentally by Zhang (2016) in terms of stress, strain and permeability evolutions and, more importantly, the transition from a flow regime determined by the intact rock matrix permeability to a flow regime determined by the crack network connectivity. The inherent capability of the model to explicitly describe the nucleation, propagation, and coalescence of stress-induced cracks, provides new insights into permeability changes occurring in low permeable rocks subjected to compressive loadings. In particular, the results confirm that permeability changes are limited up to the crack damage threshold as stress-induced cracks remain unconnected while permeability increases by several orders of magnitude afterward due to the appearance of a discrete shear band across the sample.

By conducting triaxial test simulations under different confining pressures, we found that the post-failure permeability of low permeable rocks decreases as the residual mean stress increases, and we propose exponential relationships to fit the evolution. These relationships constitutes a first attempt to characterize postfailure permeability of low permeable rocks as a function of the stress level and could thus be of interest for mitigating groundwater movements in different geoengineering contexts. Possible extensions of the present study include the introduction of pre-existing cracks in the simulated medium to better reproduce permeability reduction due to crack closure during the initial stages of the loading, investigation of hydraulically induced damage due to pore pressure variations and associated permeability changes in stressed environments, and, in the long term, implementation of multi-physics couplings to account for the effect of temperature and pressure on permeability variation.

Funding This research was funded by Agence Nationale de la Recherche, "Towards a better assessment of hydraulically induced damage in geo-engineering applications - HydroGeoDam" project, grant number ANR-17-CE06-0016.

\section{References}

Ababou R, Valera IC, Poutrel A (2011) Macro-permeability distribution and anisotropy in a 3D fissured and fractured clay rock: 'Excavation Damaged Zone'around a cylindrical drift in Callovo-Oxfordian Argilite (Bure). Physics and Chemistry of the Earth, Parts A/B/C, 36(17-18):1932-1948. https://doi.org/10.1016/j.pce.2011.07.032 
Armand G, Leveau F, Nussbaum C et al. (2014) Geometry and Properties of the Excavation-Induced Fractures at the Meuse/Haute-Marne URL Drifts. Rock Mechanics and Rock Engineering, 47(1):21-41. https://doi.org/10.1007/s00603012-0339-6

Arson C, Pereira JM (2013) Influence of damage on pore size distribution and permeability of rocks. International Journal for Numerical and Analytical Methods in Geomechanics, 37(8):810-831. https://doi.org/10.1002/nag.1123

Bogdanov II, Mourzenko VV, Thovert JF, Adler PM (2003) Effective permeability of fractured porous media in steady state flow. Water Resources Research, 39(1), 1023. https://doi.org/10.1029/2001WR000756

Bonnelye A, Schubnel A, David C, Henry P, Guglielmi Y, Gout C, Fauchille AL, Dick P (2017) Elastic wave velocity evolution of shales deformed under uppermost crustal conditions. Journal of Geophysical Research: Solid Earth, 122(1):130-141. https://doi.org/10.1002/2016JB013540

Bossart P, Meier PM, Moeri A, Trick T, Mayor JC (2002) Geological and hydraulic characterisation of the excavation disturbed zone in the Opalinus Clay of the Mont Terri Rock Laboratory. Engineering Geology, 66(1-2):19-38. https://doi.org/10.1016/S0013-7952(01)00140-5

Brace WF, Walsh JB, Frangos WT (1968) Permeability of granite under high pressure. Journal of Geophysical Research, 73(6):2225-2236. https://doi.org/10.1029/JB073i006p02225

Brace WF (1978) A note on permeability changes in geologic material due to stress. Pure and applied geophysics 116(1978):627-633. https://doi.org/10.1007/BF00876529

Brace WF (1980) Permeability of crystalline and argillaceous rocks. International Journal of Rock Mechanics and Mining Sciences \& Geomechanics Abstracts, 17(5):241-251. https://doi.org/10.1016/0148-9062(80)90807-4

Carey JW, Lei Z, Rougier E, Mori H, Viswanathan H (2015) Fracture-permeability behavior of shale. Journal of Unconventional Oil and Gas Resources, 11:27-43. https://doi.org/10.1016/j.juogr.2015.04.003

Chareyre B, Cortis A, Catalano E, Barthélemy E (2011) Pore-scale modeling of viscous flow and induced forces in dense sphere packings. Transport in Porous Media, 92(2):473-493. https://doi.org/10.1007/s11242-012-0057-2

Chen L, Liu JF, Wang CP, Liu J, Su R, Wang J (2014) Characterization of damage evolution in granite under compressive stress condition and its effect on permeability. International Journal of Rock Mechanics and Mining Sciences, 71:340-349. https://doi.org/10.1016/j.ijrmms.2014.07.020

Chen D, Pan Z, Ye Z (2015) Dependence of gas shale fracture permeability on effective stress and reservoir pressure: Model match and insights. Fuel, 139:383-392. https://doi.org/10.1016/j.fuel.2014.09.018

Chen L, Hyman JD, Lei Z, Min T, Kang Q, Rougier E, Viswanathan H (2018) Effect of fracture density on effective permeability of matrix-fracture system in shale formations. In Geological Carbon Storage (eds S Vialle, J Ajo-Franklin and JW Carey). https://doi.org/10.1002/9781119118657.ch6

Cui A, Wust R, Nassichuk B, Glover K, Brezovski R, Twemlow C (2013) A nearly complete characterization of permeability to hydrocarbon gas and liquid for unconventional reservoirs: A Challenge to Conventional Thinking. Unconventional Resources Technology Conference, Denver, Colorado, 12-14 August 2013. https://doi.org/10.1190/urtec2013-176

Cundall PA, Strack ODL (1979) A discrete numerical model for granular assemblies. Géotechnique, 29(1):47-65. https://doi.org/10.1680/geot.1979.29.1.47

Dahm T, Cesca S, Hainzl S, Braun T, Krüger F (2015) Discrimination between induced, triggered, and natural earthquakes close to hydrocarbon reservoirs: A probabilistic approach based on the modeling of depletion-induced stress changes and seismological source parameters. Journal of Geophysical Research: Solid Earth, 120(4):2491-2509. https://doi.org/10.1002/2014JB011778 
Dinç Ö, Scholtès L (2018) Discrete analysis of damage and shear banding in argillaceous rocks. Rock Mechanics and Rock Engineering, 51(5):1521-1538. https://doi.org/10.1007/s00603-017-1397-6

Dong JJ, Hsu JY, Wu WJ, Shimamoto T, Hung JH, Yeh EC, ... Sone H (2010) Stress-dependence of the permeability and porosity of sandstone and shale from TCDP Hole-A. International Journal of Rock Mechanics and Mining Sciences, 47(7):1141-1157. https://doi.org/10.1016/j.ijrmms.2010.06.019

Duriez J, Scholtès L, Donzé FV (2016) Micromechanics of wing crack propagation for different flaw properties. Engineering Fracture Mechanics, 153:378-398. https://doi.org/10.1016/j.engfracmech.2015.12.034

Frash LP, Carey JW, Lei Z, Rougier E, Ickes T, Viswanathan HS (2016) High-stress triaxial direct-shear fracturing of Utica shale and in situ X-ray microtomography with permeability measurement. Journal of Geophysical Research: Solid Earth, 121:5493-5508. https://doi.org/10.1002/2016JB012850

Guéguen Y, Gavrilenko P, Le Ravalec M (1996) Scales of rock permeability. Surveys in Geophysics, 17(3):245-263. https://doi.org/10.1007/BF01904043

Han J, Sookprasong A, Hurt R (2015) Stress field change due to reservoir depletion and its impact on refrac treatment design and SRV in shale reservoirs. SEG Global Meeting Abstracts: 2198-2208. https://doi.org/10.15530/urtec-20152144941

Hyman JD, Karra S, Carey JW, Gable CW, Viswanathan H, Rougier E, Lei Z (2018) Discontinuities in effective permeability due to fracture percolation. Mechanics of Materials, 119:25-33. https://doi.org/10.1016/j.mechmat.2018.01.005

Jafari A, Babadagli T (2013) Relationship between percolation-fractal properties and permeability of 2-D fracture networks. International Journal of Rock Mechanics and Mining Sciences, 60:353-362. https://doi.org/10.1016/j.ijrmms.2013.01.007

Jiang T, Shao JF, Xu WY, Zhou CB (2010) Experimental investigation and micromechanical analysis of damage and permeability variation in brittle rocks. International Journal of Rock Mechanics and Mining Sciences, 47(5):703-713. https://doi.org/10.1016/j.ijrmms.2010.05.003

Klinkenberg LJ (1941) The permeability of porous media to liquids and gases. Paper presented at the Drilling and production practice, New York. https://onepetro.org/APIDPP/proceedings-pdf/API41/All-API41/API-41200/2065731/api-41-200.pdf

Lemieux JM (2011) Review: The potential impact of underground geological storage of carbon dioxide in deep saline aquifers on shallow groundwater resources. Hydrogeology Journal, 19(4):757-778. https://doi.org/10.1007/s10040-011$\underline{0715-4}$

Lough MF, Lee SH, Kamath J (1998) An efficient boundary integral formulation for flow through fractured porous media. Journal of Computational Physics, 143(2):462-483. https://doi.org/10.1006/jcph.1998.5858

Massart TJ, Selvadurai APS (2014) Computational modelling of crack-induced permeability evolution in granite with dilatant cracks. International journal of rock mechanics and mining sciences, 70:593-604. https://doi.org/10.1016/j.ijrmms.2014.06.006

Matray JM, Savoye S, Cabrera J (2007) Desaturation and structure relationships around drifts excavated in the wellcompacted Tournemire's argillite (Aveyron, France). Engineering Geology, 90(1-2):1-16. https://doi.org/10.1016/j.enggeo.2006.09.021

Melin S (1989) Why are crack paths in concrete and mortar different from those in PMMA? Materials and Structures, 22(1):23-27. https://doi.org/10.1007/BF02472691

Mitchell TM, Faulkner DR (2012) Towards quantifying the matrix permeability of fault damage zones in low porosity rocks. Earth and Planetary Science Letters, 339-340:24-31. https://doi.org/10.1016/j.epsl.2012.05.014 
Moore DE, Lockner DA, Byerlee JD (1994) Reduction of permeability in granite at elevated temperatures. Science, 265(5178):1558-1561. https://doi.org/10.1126/science.265.5178.1558

Nguyen TTN, Vu MN, Tran NH, Dao NH, Pham DT (2020) Stress induced permeability changes in brittle fractured porous rock. International Journal of Rock Mechanics and Mining Sciences, 127:104224. https://doi.org/10.1016/j.ijrmms.2020.104224

Papachristos E, Scholtès L, Donzé FV, Chareyre B (2017) Intensity and volumetric characterizations of hydraulically driven fractures by hydro-mechanical simulations. International Journal of Rock Mechanics and Mining Sciences, 93:163178. https://doi.org/10.1016/j.ijrmms.2017.01.011

Paterson MS, Wong TF (2005) Experimental rock deformation - The brittle field. Springer, Berlin. https://doi.org/10.1007/b137431

Rong G, Peng J, Wang X, Liu G, Hou D (2013) Permeability tensor and representative elementary volume of fractured rock masses. Hydrogeology Journal, 21:1655-1671. https://doi.org/10.1007/s10040-013-1040-X

Sahimi M (1994) Applications of percolation theory. Taylor and Francis, London. https://doi.org/10.1201/9781482272444

Scholtès L, Donzé FV (2013) A DEM model for soft and hard rocks: Role of grain interlocking on strength. Journal of the Mechanics and Physics of Solids, 61(2):352-369. https://doi.org/10.1016/j.jmps.2012.10.005

Selvadurai APS, Głowacki A (2017) Stress-induced permeability alterations in an argillaceous limestone. Rock Mechanics and Rock Engineering, 50:1079-1096. https://doi.org/10.1007/s00603-016-1153-3

Shao JF, Zhou H, Chau KT (2005) Coupling between anisotropic damage and permeability variation in brittle rocks. International Journal for Numerical and Analytical Methods in Geomechanics, 29(12):1231-1247. https://doi.org/10.1002/nag.457

Sheng G, Su Y, Wang W (2019) A new fractal approach for describing induced-fracture porosity/permeability/ compressibility in stimulated unconventional reservoirs. Journal of Petroleum Science and Engineering, 179:855-866. https://doi.org/10.1016/j.petrol.2019.04.104

Simpson GDH, Guéguen Y, Schneider F (2001) Permeability enhancement due to microcrack dilatancy in the damage regime. Journal of Geophysical Research: Solid Earth, 106(B3):3999-4016. https://doi.org/10.1029/2000JB900194

Simpson GDH, Guéguen Y, Schneider F (2003) Analytical model for permeability evolution in microcracking rock. Pure and Applied Geophysics, 160(5):999-1008. https://doi.org/10.1007/PL00012578

Souley M, Homand F, Pepa S, Hoxha D (2001) Damage-induced permeability changes in granite: a case example at the URL in Canada. International Journal of Rock Mechanics and Mining Sciences, 38(2):297-310. https://doi.org/10.1016/S1365-1609(01)00002-8

Stormont JC, Daemen JJK (1992) Laboratory study of gas permeability changes in rock salt during deformation. International Journal of Rock Mechanics and Mining Sciences \& Geomechanics Abstracts, 29(4):325-342. https://doi.org/10.1016/0148-9062(92)90510-7

Tan X, Konietzky H (2019) Numerical simulation of permeability evolution during progressive failure of Aue granite at the grain scale level. Computers and Geotechnics, 112:185-196. https://doi.org/10.1016/j.compgeo.2019.04.016

Tanikawa W, Shimamoto T (2006) Klinkenberg effect for gas permeability and its comparison to water permeability for porous sedimentary rocks. Hydrology and Earth System Sciences Discussions, 3(4):1315-1338. https://doi.org/10.5194/hessd-3-1315-2006

Tsang CF, Birkholzer J, Rutqvist J (2007) A comparative review of hydrologic issues involved in geologic storage of CO2 and injection disposal of liquid waste. Environmental Geology, 54(8):1723-1737. https://doi.org/10.1007/s00254-007$\underline{0949-6}$ 
Tsang CF, Neretnieks I, Tsang Y (2015) Hydrologic issues associated with nuclear waste repositories. Water Resources Research, 51(9):6923-6972. https://doi.org/10.1002/2015WR017641

Yao C, Jiang QH, Shao JF (2015) A numerical analysis of permeability evolution in rocks with multiple fractures. Transport in Porous Media, 108(2):289-311. https://doi.org/10.1007/s11242-015-0476-y

Yazdi A, Hamzehpour H, Sahimi M (2011) Permeability, porosity, and percolation properties of two-dimensional disordered fracture networks. Physical Review E, 84(4). https://doi.org/10.1103/PhysRevE.84.046317

Ye Z, Ghassemi A, Riley S (2017) Laboratory investigation of fluid flow and permeability evolution through shale fractures. Proceedings of the 5th Unconventional Resources Technology Conference. https://doi.org/10.15530/urtec-2017$\underline{2674846}$

Yuan SC, Harrison JP (2006) A review of the state of the art in modelling progressive mechanical breakdown and associated fluid flow in intact heterogeneous rocks. International Journal of Rock Mechanics and Mining Sciences, 43(7):1001-1022. https://doi.org/10.1016/j.ijrmms.2006.03.004

Zhang X, Sanderson DJ (1998) Numerical study of critical behaviour of deformation and permeability of fractured rock masses. Marine and Petroleum Geology, 15(6):535-548. https://doi.org/10.1016/S0264-8172(98)00030-0

Zhang J, Standifird WB, Roegiers JC, Zhang Y (2006) Stress-dependent fluid flow and permeability in fractured media: from lab experiments to engineering applications. Rock Mechanics and Rock Engineering, 40(1):3-21. https://doi.org/10.1007/s00603-006-0103-x

Zhang CL (2016) The stress-strain-permeability behavior of clay rock during damage and recompaction. Journal of Rock Mechanics and Geotechnical Engineering, 8(1):16-26. https://doi.org/10.1016/j.jrmge.2015.10.001

Zhu W, Wong T (1997) The transition from brittle faulting to cataclastic flow: permeability evolution. J Geophys Res, 102(B2):3027-3041. https://doi.org/10.1029/96JB03282

Zimmerman RW, Bodvarsson GS (1994) Hydraulic conductivity of rock fractures. Transport in Porous Media, 23:1-30. https://doi.org/10.1007/BF00145263

Zoback MD, Byerlee JD (1975a) The effect of microcrack dilatancy on the permeability of westerly granite. Journal of Geophysical Research, 80(5):752-755. https://doi.org/10.1029/JB080i005p00752

Zoback MD, \& Byerlee JD (1975b) Permeability and effective stress. AAPG Bulletin, 59(1):154-158. https://doi.org/10.1306/83D91C40-16C7-11D7-8645000102C1865D

\section{Appendix A}

\section{A.1 Crack density and permeability}

The relationship between crack density and permeability is investigated based on the triaxial compression simulation performed under $1 \mathrm{MPa}$ confining pressure discussed in the Sect. 3.2. Besides the permeability measured in the axial $\mathrm{Y}$ direction, the permeability for fluid flow in the $\mathrm{X}$ and $\mathrm{Z}$ directions were also sequentially measured. The analysis focuses on the evolutions of permeability tensor $k x, k y$ and $k z$ with respect to the quantitative characteristics of the induced microcracks, namely the fracture intensity $P_{32}$ and the fracture volume $P_{33}$, see Fig. 13a-b. A parameter, named the average local fracture aperture $P_{33} / P_{32}$ is also represented. Since no microcracks are generated during stage $0-1$ for which $P_{33}$ equals 0 , only the points 2,3 
and 4 presented in Fig. 9a are marked on the curve of the average local fracture aperture with the log scale (Fig. $13 \mathrm{c})$.
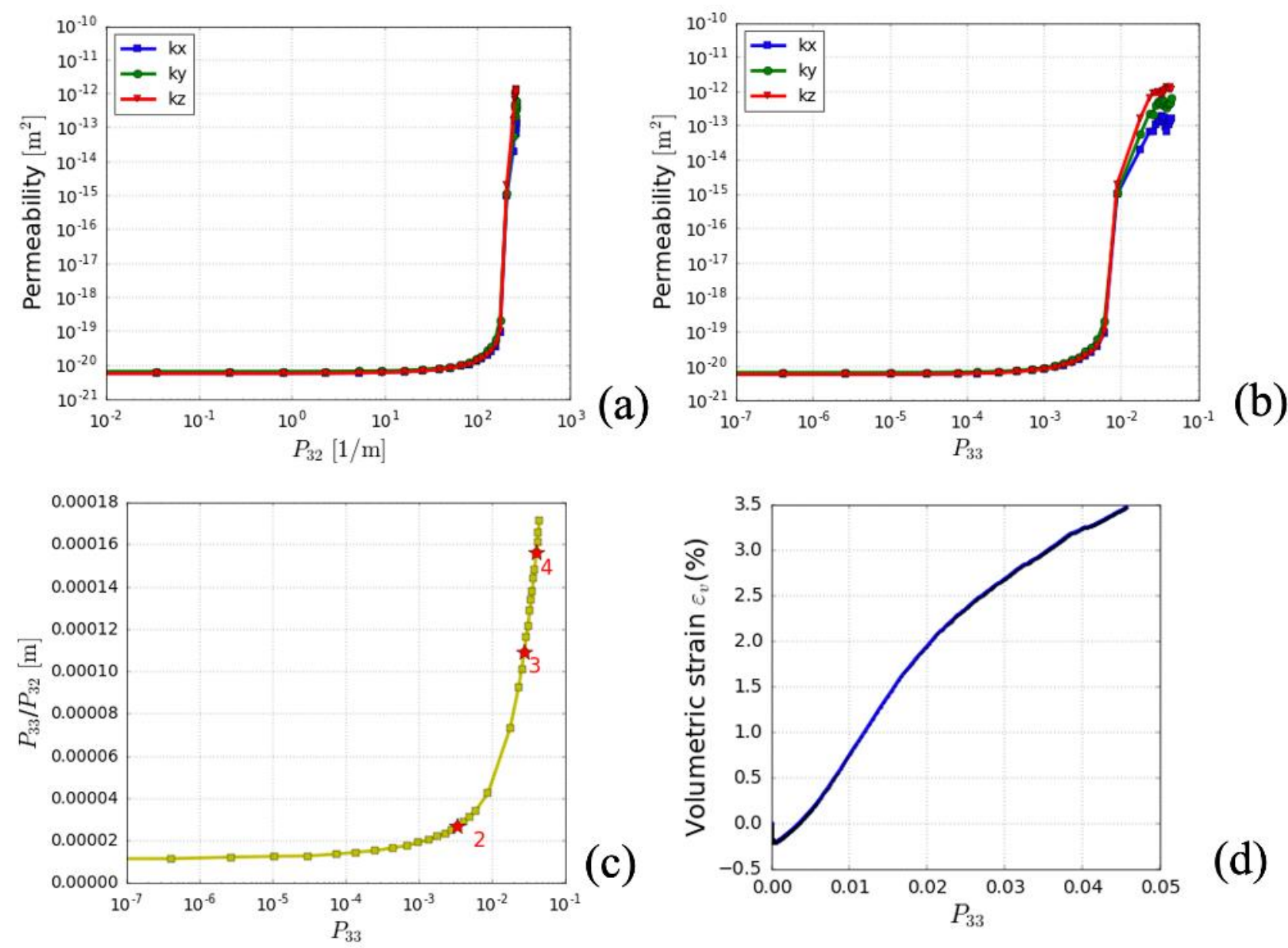

(d)

Fig. 13 Evolutions of the permeability tensor components during triaxial compression test simulations performed under a confining pressure of $1 \mathrm{MPa}$ as functions of (a) the fracture intensity $P_{32}$ and (b) the fracture volume $P_{33}$. (c) Evolution of the average local fracture aperture $P_{33} / P_{32}$ as a function of $P_{33}$, the points corresponding to loading stage 2, 3 and 4 of Fig. 9 a are marked by red stars. (d) Evolution of the volumetric strain as a function of $P_{33}$.

According to Fig. 13, before point 2, both $P_{32}$ and $P_{33}$ increases by several orders of magnitude whereas the average local fracture aperture $P_{33} / P_{32}$ only increases slightly, which indicates that the generated cracks are not yet opened. From point 2 till the end of the simulation indicated by point 4 , the significant increase of $P_{33} / P_{32}$ indicates that the cracks open more significantly. According to Fig. 13a-b, the increase of $P_{32}$ after point 2 is less important than that of $P_{33}$, therefore, the opening of crack seems to be more critical than the generation of the new cracks in terms of permeability changes. At the sample scale, a positive correlation is obtained between the volumetric strain $\epsilon_{v}$ and the fracture volume $P_{33}$ : the increase of $P_{33}$ is associated with the increase of $\epsilon_{v}$ which results from dilatancy. Furthermore, according to Fig. 10a, strain localizes in the form of a shear band that crosses the whole sample in the strain softening phase. It is thus reasonable to believe that crack opening after point 2 is more concentrated within the shear band than outside. Besides, same as indicated in Zoback et al. (1975), most of the generated cracks are orientated along with the axial stress direction. 
Therefore, the significant increase of the permeability is not only related to the coalescence of the fractures, but also to the opening of the fractures within the shear band.

According to Fig. 13, the evolutions of the permeability tensor $k x, k y$ and $k z$ generally follow the same trend. Their values are identical before point 2 , indicating that the initial sample is homogeneous. $k x, k y$ and $\mathrm{kz}$ all increase significantly by several orders of magnitude from the transition point, due to the coalescence of induced cracks. However, discrepancies exist for the three post-failure permeabilities. This discrepancy could be related to the different connectivity of the shear band to the boundaries in different directions (see Appendix A.2).

\section{A.2 Permeability tensor and strain localization}

The influence of the connectivity of the strain localization on the permeability tensor is investigated. Three samples of different sample aspect ratios, 2.5, 3 and 2, were prepared (Fig. 14a-c). The dimensions of the three samples are $7 \mathrm{~mm} \times 17 \mathrm{~mm} \times 7 \mathrm{~mm}, 7 \mathrm{~mm} \times 21 \mathrm{~mm} \times 7 \mathrm{~mm}$ and $7 \mathrm{~mm} \times 14 \mathrm{~mm} \times 7 \mathrm{~mm}$, respectively. All three samples were generated considering the same resolution with respectively 25,000, 30,000 and 20,000 particles. The evolutions of the stress-strain-permeability tensors are investigated on these three samples. The parameters as well as the mechanical and hydraulic loading conditions are the same as that of the reference simulation presented in the Sect. 3 .
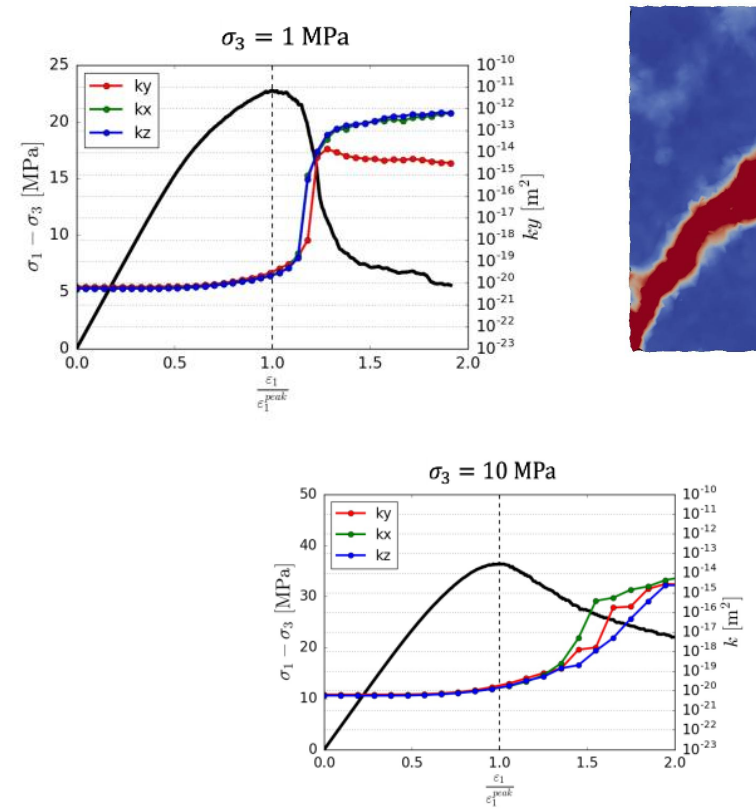

(a)
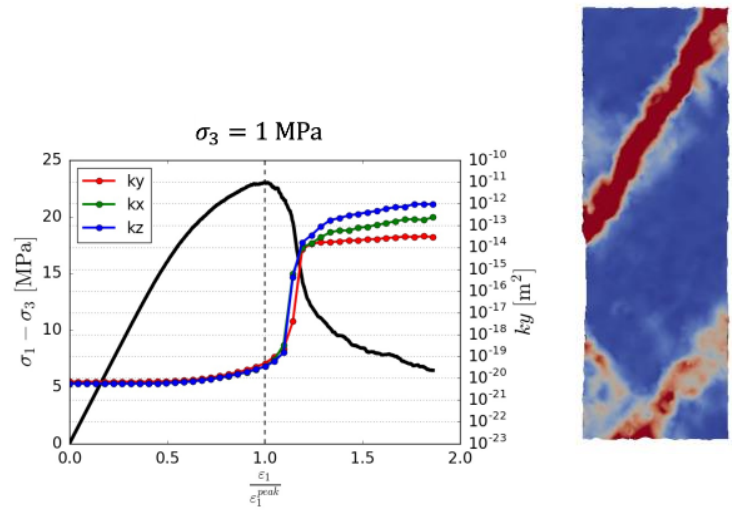

(b)

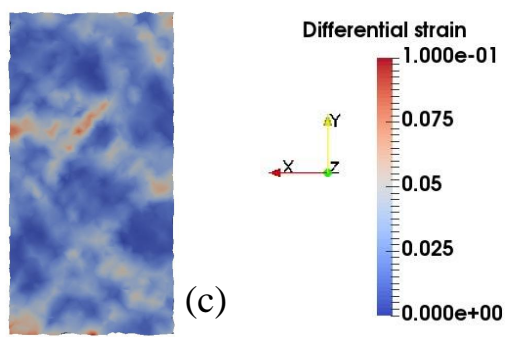

Fig. 14 Evolutions of the stress-strain-permeability tensor components during triaxial compressions test simulations performed on samples with different length to width ratios under different confining pressures and associated cumulative differential strain maps: (a) $\sigma_{3}=1 \mathrm{MPa}$, the sample aspect ratio is 2.5 . (b) $\sigma_{3}=1 \mathrm{MPa}$, the sample aspect ratio is 3. (c) $\sigma_{3}=10 \mathrm{MPa}$, the sample aspect ratio is 2. The values of the axial strain $\varepsilon_{1}$ are normalized by the axial strain at the peak stress $\varepsilon_{1}^{\text {peak }}$. 
For the first two samples, although the stress-strain responses of both samples are similar, the permeability tensors are slightly different. For the first sample presented in Fig. 14a, the post-failure permeability in the Y direction is much smaller than that in the $\mathrm{X}$ and $\mathrm{Z}$ directions. These discrepancies are related to the geometry of the shear band. Particularly, the differential strain map shown in Fig. 14a indicates that the shear band crossed the sample in the $\mathrm{X}$ and $\mathrm{Z}$ directions but not in the $\mathrm{Y}$ direction. For the second sample, the post-failure permeability is maximum for the $\mathrm{Z}$ direction and minimum for the $\mathrm{Y}$ direction, with $k x$ in between. The differences can also be related to the different connectivity of the shear band to the boundaries in different directions. According to the differential strain field presented in Fig. 14b, several shear bands have been formed within the sample. Since the shear bands completely cross the whole sample in the $\mathrm{Z}$ direction, the permeability in the $\mathrm{Z}$ direction is maximum. On the other hand, as the shear bands do not fully connect to the boundaries in the $\mathrm{Y}$ direction, the permeability is much smaller. The maximum discrepancies of the post-failure permeabilities are 1.5 order of magnitude.

For the third test conducted under $10 \mathrm{MPa}$ of confining pressure indicated in Fig. 14c, the peak value of the differential stress is much larger than for in the other two tests, whereas the post-peak stress-strain behavior is less brittle and the differential strain is less localized and its value globally smaller. The permeability values are quite homogeneous in the $\mathrm{X}, \mathrm{Y}$ and $\mathrm{Z}$ directions, which might be related to the fact that no clear shear bands have developed within the sample. This investigation highlights the important effects of the geometry of the shear bands on the anisotropy of the post-failure permeabilities.

\section{Appendix B}

\section{B.1 Effects of shear cracks on the permeability}

As mentioned in the Sect. 2, different options are available to account for the effects of tensile and shear crack on the permeability enhancement in the model. The aim here is to provide a comparison between two different options, named case (I) and case (II), to verify which option is more suitable to reproduce the experimental observations. Case (I) corresponds to the case where only tensile cracks have an effect on the permeability enhancement, as assumed in the Sect. 3. For case (II), the effects of both tensile cracks and shear cracks on the permeability enhancement are taken into account.

The stress-strain-permeability responses of the sample subjected to a triaxial compression under $1 \mathrm{MPa}$ confining pressure for both case (I) and case (II) are presented in Fig. 15. The model parameters and loading conditions are the same as that used in the Sect. 3.2. As in the experiments, both case (I) and case (II) are able to reproduce the rapid increase of permeability from the peak stress. The permeability increases by several orders of magnitude during strain softening, and the initial decrease of permeability is not reproduced in both 
cases. The permeability values of case (I) and case (II) are identical before the peak stress, whereas they show discrepancies after failure. As expected, the post-failure permeability of case (II) is one order of magnitude larger than that of case (I). Although the number of the shear cracks represents $20 \%$ of the number of tensile cracks (Fig. 11e), their conductance has an important influence on the post-failure permeability.

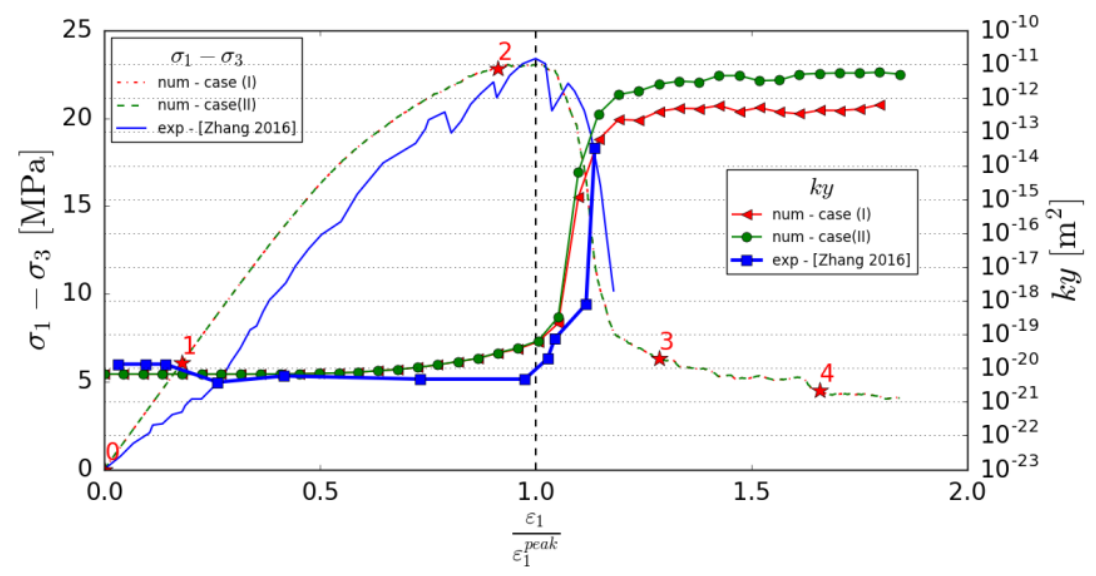

Fig. 15 Stress-strain-permeability evolution of an argillaceous rock sample (Callovo-Oxfordian clayrock) subjected to a triaxial compression performed under a confining pressure of $1 \mathrm{MPa}$ : comparison between case I (shear cracks are not considered in the flow scheme) and case II (shear cracks are considered in the flow scheme) as well as with the experimental results from Zhang (2016). The values of the axial strain $\varepsilon_{1}$ are normalized by the axial strain at the peak stress $\varepsilon_{1}^{\text {peak }}$.

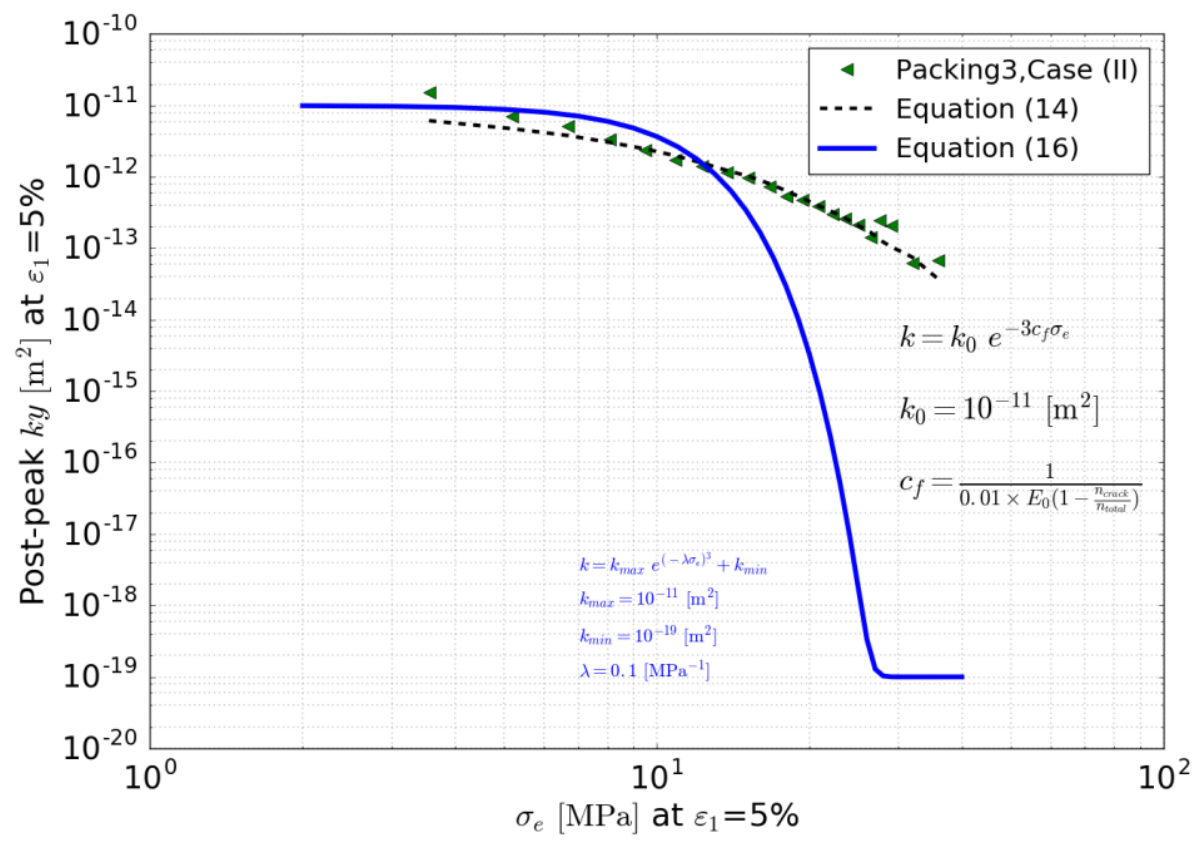

Fig. 16 Evolution of the residual (post-failure) permeability $(k y)$ as a function of the residual mean stress for simulations conducted under different confining pressures (case II). Both the permeability and the mean stress were measured for axial strain equals to $5 \%$. The solid blue line corresponds to Eq. (16) with fitted parameters mentioned in Sect. 3.3.

The relationship between the post-peak permeability and the residual mean stress under different confining pressures for the case (II) is also investigated (Fig. 16). Generally, for the case (II) the post-failure permeability decreases as the increases of the residual mean stress (which is related to the increase in confining pressure) following an exponential function. As expected, the declining rate for case (II) is much smaller than that of case 
(I). The data of case (II) can be fitted by Eq. (14): $A$ is $0.01, k_{\max }$ is $1.0 \times 10^{-11} \mathrm{~m}^{2}$ and $E_{0}$ is $4 \mathrm{GPa}$. This is because, first of all, the enhancement of permeability due to shear crack openings in case (II) is taken into account, as the local conductance for facets containing shear cracks calculated in case (II) is based on the parallel plate model, the resulting local conductance is much larger than that of the case (I); Second, the proportion of shear cracks to the total number of cracks increases when the confining pressure increases (Fig. 11e). Generally, case (I) allows to better reproduce post-failure permeability values measured in Zhang (2016). The consideration or disregard of shear crack on the permeability induce different post-failure permeabilities.

\section{B.2 Differences between gas permeability and liquid permeability}

In the literature, both liquids and gases have been used to measure permeability of porous materials. However, liquids sometimes alter the internal geometry of the porous medium and therefore its permeability. Particularly, injection of water in materials containing clay minerals leads to a decrease of permeability due to their swelling (Tsang et al. 2015). The permeability of the claystone tested in Zhang (2016) was measured with nitrogen gas. In such case, the so-called Klinkenberg effect might exist. Klinkenberg (1941) discovered that permeability to gas is relatively higher than that to water, and he interpreted this phenomenon as "slip flow" between gas molecules and solid walls. Gas molecules collide with each other and with the pore-walls when traveling through the porous medium. When the pore radius approaches the mean free path of gas molecules, the frequency of collision between gas molecules and solid walls increases. Therefore, this additional flux due to the gas flow at the wall surface, which is called "slip flow", becomes effective and tends to enhance the flow rate. This phenomenon is called Klinkenberg effect. The resulted difference between gas and liquid permeability increases with decreasing permeability (Tanikawa and Shimamoto, 2006). Correction of gas permeabilities to estimate liquid permeabilities is possible, since it was found that for a given porous medium as the mean pressure increased the measured gas permeability decreased. Unfortunately, not enough information was provided in Zhang (2016) to allow the correction of gas permeability based on Klinkenberg effect to estimate the liquid permeability.

According to the study of Dong et al. (2010), the discrepancy between the measured gas permeability and the estimated liquid permeability using the Klinkenberg correction for both sandstone and silty-shale under hydrostatic loading conditions is less than one order of magnitude. For the silty-shale samples, the hydrostatic confining pressures in the experiments varied from 3 to $120 \mathrm{MPa}$, and the measured gas permeabilities were in the range of $10^{-14}$ to $10^{-18} \mathrm{~m}^{2}$. Indeed, the Klinkenberg effect might be important in Zhang (2016) when the permeability is small, for instance before the coalescence of the micro-fractures. For triaxial loading under lower confining pressures, the Klinkenberg effect on the permeability measurement is believed to be highly reduced after the fracture coalescence, due to the opening of the microfractures within the shear band which serves as a main channel for fluid flow, the free path of the gas molecules may become smaller than the pore sizes. However, for triaxial loading conducted under larger confining pressures, since the value of the postfailure permeability is relatively close to that of the intact sample, the Klinkenberg effect might not be negligible. 


\section{Appendix C}

\section{C.1 Power-law relationships between permeability and crack density}

Supplementary information of the investigations of the Sect. 2.3.2 is provided here. The model setup is the same as that of the Sect. 2.3.2. For the comprehensive investigation of the power-law relationship between permeability and crack density for pure hydraulic loading conditions, parametric studies are conducted for two parameters, namely the joint aperture $h_{r}\left(1 \times 10^{-6} \mathrm{~m}\right.$ and $\left.2 \times 10^{-6} \mathrm{~m}\right)$ and the joint orientation $\beta\left(0^{\circ}\right.$ and $\left.90^{\circ}\right)$. The evolutions of permeability $k x, k y$ and $k z$ as a function of $P_{33}$ for three different conditions are shown in Fig. 17. The results indicate that generally the larger the fracture volume $\left(P_{33}\right)$, the larger the permeability values.
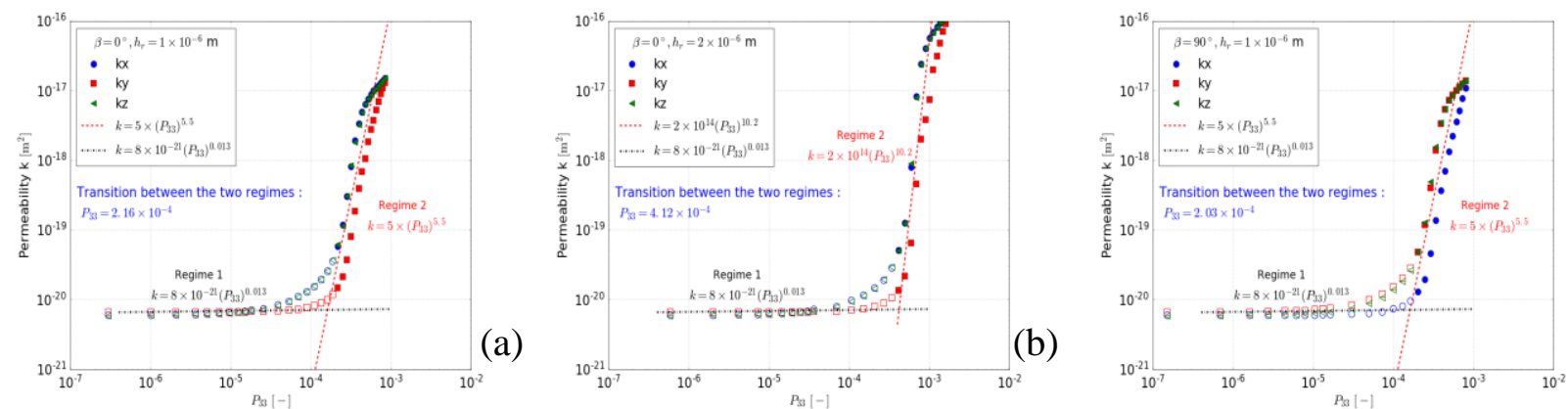

Fig. 17 Evolutions of the permeability tensor components ( $k x$ in the $\mathrm{X}$ direction, $k y$ in the $\mathrm{Y}$ direction and $k z$ in the $\mathrm{Z}$ direction) obtained with the numerical model as functions of the fracture volume $P_{33}$. Permeability values that are associated with the second power-law are represented by filled markers, otherwise they are represented by empty markers. Colors indicate the principal direction of flow, X: blue, Y: red, and Z: green. (a) $\beta=0^{\circ}, h_{r}=1 \times 10^{-6} \mathrm{~m}, \log _{10}\left(\frac{k_{f}}{k_{m}}\right)=6.8$. (b) $\beta=0^{\circ}, h_{r}=2 \times 10^{-6} \mathrm{~m}, \log _{10}\left(\frac{k_{f}}{k_{m}}\right)=$ 7.4. (c) $\beta=90^{\circ}, h_{r}=1 \times 10^{-6} \mathrm{~m}, \log _{10}\left(\frac{k_{f}}{k_{m}}\right)=6.8$.

According to Fig. 17a-b, larger hydraulic aperture induces larger values of permeability increases, and larger slope for the second power law (10.2 compared to 5.5). The transition points of the two regimes for both cases correspond to $\Delta \beta$ equals $33^{\circ}$. For bedding plane $\beta=0^{\circ}$ (Fig. 17a), the permeability in the $\mathrm{X}$ direction $k x$ is generally larger than $k y$, due to the fact that the fracture aperture after contact reorientation is proportional to the direction of the $\mathrm{X}$ flow. For bedding plane $\beta=90^{\circ}$ (Fig. 17c), opposite trends $(k y>k x)$ have been observed, as expected. When $\Delta \beta=90^{\circ}$, all the contacts are detected as joint contacts, therefore, $k x=k y=$ $k z$. As the fracture aperture always proportional to the $\mathrm{Z}$ direction, so $k z$ always equals the larger value between $k x$ and $k y$. 